\title{
The Perception and Disposal Practices of Unused And Expired Medicines By Households In an Urban Municipality, Southwest Nigeria: A Comparative Cross-Sectional Study.
}

Olufemi O. Aluko ( $D$ ooaluko@gmail.com )

Obafemi Awolowo University https://orcid.org/0000-0002-5726-5806

\section{G. T Imbianozor}

Obafemi Awolowo University Faculty of Clinical Sciences

C. O. Jideama

Obafemi Awolowo University Faculty of Clinical Sciences

O. V. Ogundele

Obafemi Awolowo University Faculty of Clinical Sciences

T. E Fapetu

Obafemi Awolowo University Faculty of Clinical Sciences

Olusegun T. Afolabi

Obafemi Awolowo University Faculty of Clinical Sciences

Research article

Keywords: Unused and expired medicines; Sound disposal legislations; Environmental protection.

Posted Date: September 24th, 2020

DOI: https://doi.org/10.21203/rs.3.rs-70874/v1

License: (-) This work is licensed under a Creative Commons Attribution 4.0 International License. Read Full License

Version of Record: A version of this preprint was published at Waste Management on March 1st, 2022. See the published version at https://doi.org/10.1016/j.wasman.2022.01.022. 


\section{Abstract}

Background: Medicines are formulations for the prevention and treatment of diseases and symptoms. Pharmaceutical wastes are products of unused, damaged, and expired medicines (UEMs) which pose a disposal challenge globally. Previous studies have shown that environmental disposal of medicines has health and environmental consequences. Hence, this study aims to determine and compare the management of household UEMs in a heterogeneous municipality in southwest Nigeria.

Methods: The study was comparative, cross-sectional in design. Multi-stage sampling identified respondents, who are females, experienced in the handling and safekeeping of medicines. The study sample was 404 and recorded a $93 \%$ response rate. Data were analysed by IBM-SPSS, version 20 . Continuous and categorical variables were presented in tables as mean $( \pm S D)$, proportions (\%) while bar charts highlight salient findings. Differences between LDS and HDS values were determined by $\chi^{2}\left(P_{a}>0.05\right)$.

Results: $53.4 \%$ and $71.2 \%$ of respondents respectively had good knowledge and positive attitudes to safe disposal of UEMs. At least $31.5 \%$ households do not stock medicines in homes while 1-2 sachets of antimalarial (57.3\%), analgesics (52.7\%) and antibiotics (49.7\%) medicines were inventoried in households and significantly different between low-density (LD) and high-density (HD) settlements. $72.9 \%$ and $67.8 \%$; 47.9\% and $55.6 \%$ respondents in LD and HD settlements respectively disposed of household solid and liquid UEMs in thrash-bins, though $34.9 \%$ (LDS) and 16.7\% (HDS) disposed of liquid UEMs in the toilet/sink. There were significant differences in medicines abundance and disposal practices between LD and HD settlements for solid and liquid medications. $37.1 \%$ of respondents perceived consequences for the current methods of disposing of UEMs, which include accidental ingestion (76.6\% vs $26.7 \%)$ and land pollution $(69.6 \%$ vs $36.7 \%)$ and water pollution $(72.4 \%$ vs $32.8 \%$ ) and drugs toxicity ( $58.3 \%$ vs $32.8 \%)$, respectively in LD and HD settlements.

Conclusions: Respondents good knowledge and positive attitudes contrasted the poor UEMs disposal practice. Most of the households' medicines were antimalaria and analgesics and there were disparities in the disposal of solid and liquid medicines, between LD and HD settlements, respectively. Appropriate legislation, compliance monitoring and enforcement are drivers to incentive-driven, sound recovery systems for UEMs to alleviate the consequences of current disposal practices in Nigeria.

\section{Background}

A drug is any substance used in the diagnosis, treatment or prevention of a disease, a component of medication or food supplement [1]. The active pharmaceutical ingredients and the target site for drugs vary with some used for chemotherapy and others with action on the nervous, cardiovascular or endocrine systems [2]. Broadly, drugs are categorized as prescription, over the counter (OTC) and off-label types. Drugs are indispensable and part of human survival strategy [3]. Globally, there is an increase in drugs production and consumption in developing and developed countries $[4,5]$, with a quantum eventually meant for disposal when expired. 
The World Health Organisation (WHO) propounded that half of the prescription drugs end up unused [6], mainly due to recovery feelings, drugs expiry, dosage regimen change and adverse effects [7]. Hospitals and healthcare service providers are the major sources of drugs and healthcare waste (HCW), though home healthcare service, early discharge after surgeries and the home management of some illnesses have made drugs and HCW abundant in the home environment [8]. In recent times, infectious and Non-communicable diseases (NCDs) have increased prescription and dispensing medications [7]. This creates concern about the handling and management of medical waste, including medicines outside the hospital environment [9]. In countries with policies on safe disposal of medicines, more than half still flushed unused medications in sewerage facilities [10] while only a minority returned unused medications to the prescribing facility [11] while in Kabul (Afghanistan) and Vienna (Austria), most respondents kept unused medication at home and disposed expired drugs into the trash at home [7, 12].

In the study area, water treatment systems and regulations do not monitor drugs residues as an indication of potability $[10,13]$ and there are pieces of evidence that improper disposal of drugs could contaminate water resources while active pharmaceutical ingredients (APIs) persists in the environmental media [8]. Also, ingestion of expired drugs that were inappropriately discarded may lead to toxicity and drug resistance. [14]. In the study area, it is a common practice to keep unused and, or expired medications in homes after discontinued use or excesses purchased for self-medication. Usually, expired drugs are not supposed to be disposed of with household refuse and as such when disposed of, may herald apparent health (upon accidental or inappropriate ingestion $[14,15]$, environmental consequences $[8,16,17]$ and antibiotic resistance $[18,19]$. The study aims to determine and compare the perception and drug management regimen among high-density and low-density households in the absence of national guidelines for the disposal of unused and expired drugs in the study area.

\section{Methods}

\section{Description Of The Study Area}

The study took place in Ife Central Local Government Area (LGA). Ile-lfe is an ancient Yoruba city and the cradle of the Yoruba race. The city is in Osun East senatorial district in the southwest of Nigeria. The LGA has 11 wards and a total of $11 \mathrm{~km}^{2}$ lies between latitudes $25^{\circ} \mathrm{N}$ and $30^{\circ} \mathrm{N}$ of the equator and bounded by IfeNorth, Ife-South, Ayedaade, Atakumosa-West and Ife-East LGAs. Geographically, lle-Ife experiences distinct rainy (April and October) and dry (November and March) seasons. The annual rainfall is about 1,330 mm with the highest temperature hovering over $28.3^{\circ} \mathrm{C}$. Many residents are farmers with about $75 \%$ of the population engaged in agriculture and agro-allied industries while others are traders and civil servants. The predominant religions are Christianity, Islam, and traditional religion. The population is 167,204 , male 88,403 and female 78,801 [20]. The LGA is densely populated by the Yoruba speaking people of southwestern Nigeria, in addition to other ethnic groups; such as Igbo and Hausa.

\section{Study Design}


The study is a comparative cross-sectional in nature and assessed the drugs handling and disposal practices of households in high-density and low-density settlements in the study area.

\section{Target and Study Population}

The study targeted all households in Ife Central LGA though studied selected households and interacted with women experienced in the drugs handling and disposal for at least one year.

\section{Inclusion And Exclusion Criteria}

Women who consented and have lived in the selected household for at least 12 months were enrolled into the study while those who were eligible consented and willing to participate in the study but incapacitated through sickness or mentally derailed were excluded from the study.

\section{Sample Size}

The study sample size for this study was calculated using the sample size formula:

Where:

$n$ per group $=\frac{\left(Z_{\alpha}+Z_{\beta}\right)^{2} * p_{1}\left(1-p_{1}\right) p_{2}\left(1-p_{2}\right)}{\left(p_{1}-p_{2}\right)^{2}}$

$\mathrm{n}_{\text {per group }}=$ sample size per group

$z_{a}=$ level of confidence

Standard normal variation for $95 \%$ confidence level, $\left(Z_{a}=1.96\right)$

$\mathrm{z}_{\mathrm{b}}=$ power of intense

Standard normal variation for $80 \%$ confidence level, $\left(Z_{b}=1.28\right)$

$p_{1}=$ prevalence of respondents that dispose of drugs with garbage $(95 \%)$ in low-density settlements, $\left(P_{1}=\right.$ $0.95[15]$.

$p_{2}=$ prevalence of respondents that dispose of drugs with garbage $(95 \%)$ in low-density settlements, $\left(P_{1}=\right.$ $0.05[15]$.

$\mathrm{q}_{1}=1-\mathrm{p}_{1}$ hence $\mathrm{q}_{1}=0.05(5 \%)$

$\mathrm{q}_{2}=1-\mathrm{p}_{2}$ hence $\mathrm{q}_{2}=0.15(15 \%)$ 
$n_{\text {per group }}=202$ with $10 \%$ added for non-response, giving a total of 404 as the study minimum sample size .

\section{Sampling Technique}

The study applied a four-stage sampling technique. In stage one: the 11 political wards were stratified into six urban and five rural groups with two Wards selected from each group by simple random sampling technique. In stage two, streets in each of the four wards were stratified into high-density and low-density settlements with simple random sampling applied to select two from each group. Due to absence of the street sampling frame, systematic sampling selected even-numbered houses in selected streets from the proximate house at the street entrance until 26 households were approached, in each of the eight streets in stage three. In houses where there were multiple eligible households, simple random sampling was used to identify responding household among those available that met the inclusion criteria in stage four.

\section{Data Collection}

The study used a pretested, interviewer-administered semi-structured questionnaire that was designed in the English Language and translated to Yoruba and back-translated into English to ensure contents validity. The study questionnaire was however adapted with permission, from [9] and administered on respondents between January and February 2018.

\section{Data Analysis}

Data entry and analysis were done using the International business machine (IBM-Statistical package for social sciences (SPSS) version 20. Discrete variables were presented with tables and bar charts while the associations among related variables were determined by chi-square and at a p-value of $<0.05$.

\section{Outcome measures}

Knowledge, attitude and perception on the handling and disposal of unused and expired drugs was a composite measure of 10, 13 and 23 variables, respectively. Correct responses were scored ' 1 ' and incorrect ' 0 '. The median (IQR) values; respectively 7(6-8), 7(7-8) and 14(13-15) for knowledge, attitude and perception were used to dichotomise $\geq$ median composite values into good/positive/high and those below the mean values as poor/negative/low ratings, respectively. The awareness of respondents on the protocol for disposal of unwholesome medicines was the binary, dependent variable while socio-demographic variables, including knowledge attitude, perception, and types of settlements, were independent variables in the binary logistic regression model. Similarly, the perception was a composite index of 23 variables.

\section{Ethical Considerations}

The study obtained ethical approval (IPHOAU/12/1008) from the research and ethics committee of the Institute of Public Health (IPH) of the Obafemi Awolowo University, in Nigeria. Also, permission and informed consent were respectively obtained from the head of the selected household and respondents. Responses 
were anonymised to preserve the confidentiality of respondents and personal identifiers were removed in summary data.

\section{The study strengths and weaknesses}

The study is cross-sectional in design and the respondents were randomly selected through multi-stage sampling technique to reflect their characteristics. Hence, the findings can be generalised to the study population. In contrast, the study is prone to social desirability response bias, perhaps due to serial sematic order effects [21].

\section{Results}

\section{Socio-demographic characteristics of respondents}

The study showed that the mean age of respondents in the LDS and HDS were respectively $36.1 \pm 0.6$ and $37.7 \pm 0.7$ years. Also, $82,8 \%$, and $70.6 \%$, respectively in the LDS and HDS were aged between 25 and 44 years. Most (82.5\%) of the respondents belonged to the Yoruba ethnicity, with a similar trend in LDS (74.0\%) and HDS (91.7\%). Similarly, more than four out of five respondents (82.8\%) are married, distributed as $74.0 \%$ and $92.2 \%$, respectively in LDS and HDS. The study also revealed that tertiary (50.0\%) and secondary (49.0\%) education predominates in LDS in contrast to secondary education (77.8\%) in HDS, respectively (Table 1). A higher proportion of residents in LDS are civil servant (28.1\%) when compared with those in HDS (3.9\%) while higher proportions of residents in LDS (44.3\%) and HDS (67.8\%) were self-employed. At the same time, LDS and HDS was home to $87.5 \%$ and $56.1 \%$ of residents earning, respectively above and below the national minimum wage monthly, with the mean income for respondents in the LDS and HDS being $\$ 48,164 \pm 2,896$ and $\$ 18,405 \pm 793$, respectively. Besides, the study revealed that more respondents use the water closet toilet in the LDS (76.0\%) than in the HDS (47.2\%). Further, the modal household size in both LDS and HDS was 4 and strangely, $48.4 \%$ and $42,2 \%$ were rated poor and average, wealth-wise, respectively. Also, there were significant differences in all socio-demographic characteristics of respondents, between residents in LDS and HDS. (Table 1). 
Table 1

Sociodemographic characteristics of respondents

\begin{tabular}{|c|c|c|c|c|c|c|c|c|}
\hline \multirow[t]{2}{*}{$\begin{array}{l}\text { Description of } \\
\text { variables }\end{array}$} & \multirow[t]{2}{*}{ Categories } & \multicolumn{2}{|c|}{$\begin{array}{l}\text { Low-density } \\
\text { settlement }\end{array}$} & \multicolumn{2}{|c|}{$\begin{array}{l}\text { High-density } \\
\text { settlement }\end{array}$} & \multicolumn{2}{|c|}{ Total } & \multirow[t]{2}{*}{$\begin{array}{l}\text { Test } \\
\text { statistics }\end{array}$} \\
\hline & & $\mathbf{n}$ & $\%$ & $\mathbf{n}$ & $\%$ & $\mathbf{N}$ & $\%$ & \\
\hline \multirow{3}{*}{$\begin{array}{l}\text { Age } \\
\text { (years) }\end{array}$} & $\leq 24$ & 8 & 4.2 & 10 & 5.6 & 18 & 4.8 & \multirow{3}{*}{$\begin{array}{l}\bigotimes 2= \\
8.189 \\
d f=2 ; \\
p= \\
0.017 \star\end{array}$} \\
\hline & $25-44$ & 159 & 82.8 & 127 & 70.6 & 286 & 76.9 & \\
\hline & $\geq 45$ & 25 & 13.0 & 43 & 23.9 & 68 & 18.3 & \\
\hline \multirow[t]{3}{*}{ Ethnicity } & Yoruba & 142 & 74.0 & 165 & 91.7 & 307 & 82.5 & \multirow{3}{*}{$\begin{array}{l}\otimes 2= \\
21.931 ; \\
d f=2 ; \\
P= \\
0.0001^{\star}+\end{array}$} \\
\hline & Igbo & 49 & 25.5 & 14 & 7.8 & 63 & 16.9 & \\
\hline & Others (Hausa, Fulani) & 01 & 0.5 & 01 & 0.6 & 02 & 0.5 & \\
\hline \multirow[t]{3}{*}{ Religion } & Islam & 49 & 25.5 & 72 & 40.0 & 121 & 32.5 & \multirow{3}{*}{$\begin{array}{l}\bigotimes 2= \\
11.170 ; \\
d f=2 ; \\
P= \\
0.004^{\star}+\end{array}$} \\
\hline & Christianity & 141 & 73.4 & 108 & 60.0 & 249 & 66.9 & \\
\hline & Traditional & 02 & 1.0 & 00 & 0.0 & 02 & 0.5 & \\
\hline \multirow[t]{4}{*}{ Marital Status } & Single & 19 & 9.9 & 06 & 3.3 & 25 & 6.7 & \multirow{4}{*}{$\begin{array}{l}\otimes 2= \\
25.473 \\
d f=3 \\
P= \\
0.0001^{*}+\end{array}$} \\
\hline & Single parenthood & 21 & 10.9 & 03 & 1.7 & 24 & 6.5 & \\
\hline & Married & 142 & 74.0 & 166 & 92.2 & 308 & 82.8 & \\
\hline & $\begin{array}{l}\text { Others (widowed, } \\
\text { divorced, separated) }\end{array}$ & 10 & 5.2 & 05 & 2.8 & 15 & 4.0 & \\
\hline \multirow{4}{*}{$\begin{array}{l}\text { Highest } \\
\text { education }\end{array}$} & Primary Education & 01 & 0.4 & 12 & $6 / 7$ & 13 & 3.5 & \multirow{4}{*}{$\begin{array}{l}\otimes 2= \\
67.872 ; \\
d f=3 ; \\
p= \\
0.0001^{*}+\end{array}$} \\
\hline & Secondary Education & 94 & 49.0 & 140 & 77.8 & 234 & 62.9 & \\
\hline & Tertiary Education & 96 & 50.0 & 24 & 13.3 & 20 & 32.3 & \\
\hline & Vocational & 01 & 0.5 & 04 & 2.2 & 05 & 1.3 & \\
\hline \multirow[t]{4}{*}{ Occupation } & Civil servant & 54 & $28.1 \%$ & 07 & 3.9 & 61 & 16.4 & \multirow{4}{*}{$\begin{array}{l}\otimes 2= \\
48.029 \\
d f=3 \\
p= \\
0.0001 *\end{array}$} \\
\hline & Self employed & 85 & 44.3 & 122 & 67.8 & 207 & 55.6 & \\
\hline & Paid private sector & 35 & 18.2 & 22 & 12.2 & 57 & 15.3 & \\
\hline & $\begin{array}{l}\text { Others (full-time } \\
\text { housewife, } \\
\text { unemployed) }\end{array}$ & 18 & $9.4 \%$ & 29 & 16.1 & 47 & 12.6 & \\
\hline
\end{tabular}

Key: ${ }^{*}=$ significant different between low-density and high-density settlements; $+=$ Likely ratio chi square . 


\begin{tabular}{|c|c|c|c|c|c|c|c|c|}
\hline \multirow{3}{*}{$\begin{array}{l}\text { Household } \\
\text { size }\end{array}$} & $\leq 4$ persons & 129 & 67.2 & 91 & 50.6 & 220 & 59.1 & \multirow{3}{*}{$\begin{array}{l}\otimes 2= \\
10.635 ; \\
d f=1 ; \\
p= \\
0.0001\end{array}$} \\
\hline & \multirow[t]{2}{*}{$\geq 5$ persons } & \multirow[t]{2}{*}{63} & \multirow[t]{2}{*}{32.8} & \multirow[t]{2}{*}{89} & \multirow[t]{2}{*}{49.4} & \multirow[t]{2}{*}{152} & \multirow[t]{2}{*}{40.9} & \\
\hline & & & & & & & & \\
\hline \multirow[t]{3}{*}{$\begin{array}{l}\text { Household } \\
\text { toilet type }\end{array}$} & Water closet & 146 & 76.0 & 87 & 47.2 & 231 & 62.1 & \multirow{3}{*}{$\begin{array}{l}\otimes 2= \\
33.807 ; \\
d f=2 ; \\
p= \\
0.0001 *\end{array}$} \\
\hline & Pit latrine & 44 & 22.9 & 88 & 48.9 & 132 & 35.5 & \\
\hline & None & 02 & 1.0 & 07 & 3.9 & 9 & 2.4 & \\
\hline \multirow[t]{2}{*}{$\begin{array}{l}\text { Income class } \\
\text { (\#) }\end{array}$} & $\leq \# 18,000$ & 24 & 12.5 & 101 & 56.1 & 125 & 33.6 & \multirow{2}{*}{$\begin{array}{l}\searrow 2= \\
79.196 ; \\
d f=1 ; \\
p= \\
0.0001\end{array}$} \\
\hline & $>\# 18,000$ & 168 & 87.5 & 79 & 43.9 & 247 & 66.4 & \\
\hline \multirow[t]{3}{*}{$\begin{array}{l}\text { Wealth } \\
\text { categories }\end{array}$} & Poor & 93 & 48.4 & 29 & 16.1 & 122 & 32.8 & \multirow{3}{*}{$\begin{array}{l}\llbracket 2= \\
44.065 ; \\
d f=2 ; \\
p= \\
0.0001^{*}\end{array}$} \\
\hline & Average & 49 & 25.5 & 76 & 42.2 & 125 & 33.6 & \\
\hline & Rich & 50 & 26.0 & 75 & 41.7 & 125 & 33.6 & \\
\hline
\end{tabular}

Key: ${ }^{*}=$ significant different between low-density and high-density settlements; + = Likely ratio chi square.

\section{Drugs acquisition and duration of use characteristics}

The health-seeking behaviour by respondents was healthcare facilities, made within three months of the study by a higher proportion of the study population, though not statistically significant between LDS and HDS. Also, a higher proportion of respondents, $82.2 \%$ and $68.3 \%$, respectively in LDS and HDS visit pharmacy for drugs acquisition (Table 2). The study showed that a higher proportion of respondents in LDS (95.3\%), compared with HDS (92.8\%) received drugs as part of treatment received, though $75.0 \%$ (LDS) and $91.1 \%$ (HDS) of drugs in respondent's homes were acquired through self-medication. Drugs received for what? $49.5 \%$ and $24.7 \%$ sought treatment for malaria and diarrhoea related causes. The higher proportion of respondents $(61.1 \%)$ compared to $38.5 \%$ sought treatment for malaria-related causes while more of residents in LDS (28.1\%) sought treatment for diarrhoea related ailments. The higher proportion of the sick in most homes in LDS (56.8\%) did not comply with the drug use regimen while a higher proportion (51.7\%) complied with the use regimen for prescribed medicines, within the first week of treatment. Most of those that failed to complete prescribed medications felt better in LDS (83.6\%) and HDS (93.0\%) while some stopped medications due to side effects, among others (Table 2). 
Table 2

Sources of drugs acquired and duration of use characteristics

\begin{tabular}{|c|c|c|c|c|c|c|c|c|}
\hline \multirow[t]{2}{*}{ Description of variables } & \multirow[t]{2}{*}{ Categories } & \multicolumn{2}{|c|}{$\begin{array}{l}\text { Low-density } \\
\text { settlement }\end{array}$} & \multicolumn{2}{|c|}{$\begin{array}{l}\text { High-density } \\
\text { settlement }\end{array}$} & \multicolumn{2}{|c|}{ Total } & \multirow[t]{2}{*}{$\begin{array}{l}\text { Test } \\
\text { statistics }\end{array}$} \\
\hline & & $\mathrm{n}$ & $\%$ & $\mathbf{N}$ & $\%$ & $\mathbf{N}$ & $\%$ & \\
\hline \multirow[t]{4}{*}{ Last hospital visit $(n=372)$} & $\begin{array}{l}1-3 \\
\text { months }\end{array}$ & 100 & 52.1 & 76 & 42.2 & 176 & 47.3 & \multirow{4}{*}{$\begin{array}{l}凶 2= \\
3.921 ; \\
d f=3 ; \\
p=0.270\end{array}$} \\
\hline & $\begin{array}{l}4-6 \\
\text { months }\end{array}$ & 34 & 17.7 & 39 & 21.7 & 73 & 19.6 & \\
\hline & $\begin{array}{l}7-12 \\
\text { months }\end{array}$ & 41 & 21.4 & 43 & 23.9 & 84 & 22.6 & \\
\hline & $\begin{array}{l}\geq 13 \\
\text { months }\end{array}$ & 17 & 8.9 & 22 & 12.2 & 39 & 10.5 & \\
\hline \multirow[t]{4}{*}{$\begin{array}{l}\text { Last Pharmacy visit }(n= \\
371)\end{array}$} & $\begin{array}{l}1-3 \\
\text { months }\end{array}$ & 157 & 82.2 & 123 & 68.3 & 280 & 75.5 & \multirow{4}{*}{$\begin{array}{l}凶 2= \\
10.731 \\
d f=3 ; \\
P= \\
0.013^{*}\end{array}$} \\
\hline & $\begin{array}{l}4-6 \\
\text { months }\end{array}$ & 21 & 11.0 & 34 & 18.9 & 55 & 14.8 & \\
\hline & $\begin{array}{l}7-12 \\
\text { months }\end{array}$ & 8 & 4.2 & 18 & 10.0 & 26 & 7.0 & \\
\hline & $\begin{array}{l}\geq 13 \\
\text { months }\end{array}$ & 5 & 2.6 & 5 & 2.8 & 10 & 2.7 & \\
\hline \multirow[t]{5}{*}{$\begin{array}{l}\text { Last hospital herbal } \\
\text { medicine vendor }(n=372)\end{array}$} & $\begin{array}{l}\text { Not } \\
\text { Applicable }\end{array}$ & 137 & 71.4 & 81 & 45.0 & 218 & 58.6 & \multirow{5}{*}{$\begin{array}{l}\qquad 2= \\
37.940 \\
\text { df }=4 ; \\
P= \\
0.0001 *\end{array}$} \\
\hline & $\begin{array}{l}1-3 \\
\text { months }\end{array}$ & 25 & 13.0 & 60 & 33.3 & 85 & 22.8 & \\
\hline & $\begin{array}{l}4-6 \\
\text { months }\end{array}$ & 5 & 2.6 & 18 & 10.0 & 23 & 6.2 & \\
\hline & $\begin{array}{l}7-12 \\
\text { months }\end{array}$ & 13 & 6.8 & 15 & 8.3 & 28 & 7.5 & \\
\hline & $\begin{array}{l}\geq 13 \\
\text { months }\end{array}$ & 12 & 6.2 & 6 & 3.3 & 18 & 4.8 & \\
\hline \multirow[t]{4}{*}{$\begin{array}{l}\text { Reason for seeking } \\
\text { treatment }(n=372)\end{array}$} & $\begin{array}{l}\text { malaria } \\
\text { related }\end{array}$ & 74 & 38.5 & 110 & 61.1 & 184 & 49.5 & \multirow{4}{*}{$\begin{array}{l}\llbracket 2= \\
20.797 ; \\
d f=3 \\
P= \\
0.0001 \text { * }\end{array}$} \\
\hline & $\begin{array}{l}\text { diarrhoea } \\
\text { related }\end{array}$ & 54 & 28.1 & 38 & 21.1 & 92 & 24.7 & \\
\hline & $\begin{array}{l}\text { injury and } \\
\text { swelling }\end{array}$ & 32 & 16.7 & 19 & 41.3 & 51 & 13.7 & \\
\hline & Others & 32 & 16.7 & 13 & 7.2 & 45 & 12.1 & \\
\hline
\end{tabular}




\begin{tabular}{|c|c|c|c|c|c|c|c|c|}
\hline \multirow{3}{*}{$\begin{array}{l}\text { Received drugs as a result } \\
\text { of treatment }(n=372)\end{array}$} & Yes & 183 & 95.3 & 167 & 92.8 & 350 & 94.1 & \multirow{3}{*}{$\begin{array}{l}\boxplus 2= \\
1.423 \\
d f=2 ; \\
p= \\
0.491^{++}\end{array}$} \\
\hline & No & 6 & 3.1 & 7 & 3.9 & 13 & 3.5 & \\
\hline & $\begin{array}{l}\text { I don't } \\
\text { know }\end{array}$ & 3 & 1.6 & 6 & 3.3 & 9 & 2.4 & \\
\hline \multirow[t]{3}{*}{$\begin{array}{l}\text { Drugs prescribed by }(\mathrm{n}= \\
372)\end{array}$} & Pharmacist & 0 & 0.0 & 2 & 1.1 & 2 & 0.5 & \multirow{2}{*}{$\begin{array}{l}\llbracket 2= \\
21.579 \\
d f=2\end{array}$} \\
\hline & Physician & 48 & 25.0 & 14 & 7.8 & 62 & 16.7 & \\
\hline & $\begin{array}{l}\text { Self- } \\
\text { medication }\end{array}$ & 144 & 75.0 & 164 & 91.1 & 308 & 82.8 & $\begin{array}{l}p= \\
0.0001 *\end{array}$ \\
\hline \multirow{3}{*}{$\begin{array}{l}\text { Period of drugs use }(n= \\
372)\end{array}$} & 1-7 days & 166 & 86.5 & 168 & 93.3 & 334 & 89.8 & \multirow{3}{*}{$\begin{array}{l}\boxplus 2= \\
7.340 \\
d f=2 ; \\
p= \\
0.025^{\star^{++}}\end{array}$} \\
\hline & $\geq 8$ days & 23 & 12.0 & 8 & 4.4 & 31 & 8.3 & \\
\hline & $\begin{array}{l}\text { Routine } \\
\text { use }\end{array}$ & 3 & 1.6 & 4 & 2.2 & 7 & 1.9 & \\
\hline \multirow[t]{2}{*}{$\begin{array}{l}\text { The sick completed } \\
\text { medication }(n=372)\end{array}$} & Yes & 83 & 43.2 & 93 & 51.7 & 176 & 47.3 & \multirow{2}{*}{$\begin{array}{l}\bigotimes 2= \\
2.656 \\
d f=1 ; \\
p=0.103\end{array}$} \\
\hline & No & 109 & 56.8 & 87 & 48.3 & 196 & 52.7 & \\
\hline \multirow[t]{3}{*}{$\begin{array}{l}\text { Reasons for non-completion } \\
\text { of medication }(n=196)\end{array}$} & Felt better & 92 & 83.6 & 80 & 93.0 & 172 & 87.8 & \multirow{3}{*}{$\begin{array}{l}\llbracket 2= \\
6.412 ; \\
d f=2 ; \\
p= \\
0.041^{++}\end{array}$} \\
\hline & $\begin{array}{l}\text { Side } \\
\text { effects }\end{array}$ & 9 & 8.2 & 5 & 5.8 & 14 & 7.1 & \\
\hline & Others & 9 & 8.2 & 1 & 1.2 & 10 & 5.1 & \\
\hline
\end{tabular}

Key: * = significant different between low-density and high-density settlements; ${ }^{++}=$Likely ratio chi square

\section{Knowledge of expiry and disposal of expired and unused medication}

Knowledge has been mooted as influencing attitude and practice. The knowledge of respondents on drugs expiry and disposal of UEMs is presented in Table 3. Most respondents in HDS and LDS knew that drugs are those formulated, packaged, and sold in pharmacies with at least $85.9 \%$ in LDS. The highest proportions of respondents knew that all medicines have use instructions, with $65.1 \%$ and $41.9 \%$ while more than two-fifths were not aware of medicines use prescriptions under different weights and age categories. $96.5 \%$ of respondents knew that drugs should be handled with care with a higher proportion of respondents $(96.7 \%)$ from HDS. 
Table 3

Knowledge of respondents on expiry and disposal of expired and unused medication

\begin{tabular}{|c|c|c|c|c|c|c|c|c|}
\hline \multirow[t]{2}{*}{ Description of variables } & \multirow[t]{2}{*}{ Categories } & \multicolumn{2}{|c|}{$\begin{array}{l}\text { Low- } \\
\text { density } \\
\text { settlement }\end{array}$} & \multicolumn{2}{|c|}{$\begin{array}{l}\text { High- } \\
\text { density } \\
\text { settlement }\end{array}$} & \multicolumn{2}{|c|}{ Total } & \multirow[t]{2}{*}{$\begin{array}{l}\text { Test } \\
\text { statistics }\end{array}$} \\
\hline & & $\mathbf{n}$ & $\%$ & $\mathbf{N}$ & $\%$ & $\mathrm{~N}$ & $\%$ & \\
\hline \multirow{3}{*}{$\begin{array}{l}\text { Respondents understanding } \\
\text { of what constitute a drug } \\
(n=372)\end{array}$} & $\begin{array}{l}\text { Self-made } \\
\text { herbal } \\
\text { mixtures }\end{array}$ & 18 & 9.4 & 5 & 2.8 & 23 & 6.2 & \multirow{3}{*}{$\begin{array}{l}\otimes 2= \\
7.245 \\
d f=2 ; \\
p=0.027\end{array}$} \\
\hline & $\begin{array}{l}\text { Packaged } \\
\text { herbal } \\
\text { formulations }\end{array}$ & 9 & 4.7 & 7 & 3.9 & 16 & 4.3 & \\
\hline & $\begin{array}{l}\text { Medicine } \\
\text { sold in } \\
\text { pharmacies }\end{array}$ & 165 & 85.9 & 168 & 93.3 & 333 & 89.6 & \\
\hline \multirow[t]{3}{*}{$\begin{array}{l}\text { All drugs have use } \\
\text { instructions }(n=371)\end{array}$} & Yes & 125 & 65.1 & 75 & 41.9 & 200 & 53.9 & \multirow{3}{*}{$\begin{array}{l}\varangle 2= \\
57.942 ; \\
d f=2 ; \\
p= \\
0.000 *\end{array}$} \\
\hline & No & 16 & 8.3 & 76 & 42.5 & 92 & 24.8 & \\
\hline & I don't know & 51 & 26.6 & 28 & 15.6 & 79 & 21.3 & \\
\hline \multirow{3}{*}{$\begin{array}{l}\text { Drugs should be handled with } \\
\text { care and caution }(n=372)\end{array}$} & Yes & 185 & 96.4 & 174 & 96.7 & 359 & 96.5 & \multirow{3}{*}{$\begin{array}{l}\otimes 2= \\
5.754 ; \\
d f=2 ; \\
P=0.056\end{array}$} \\
\hline & No & 0 & 0.0 & 3 & 1.7 & 3 & 0.8 & \\
\hline & I don't know & 7 & 3.6 & 3 & 1.7 & 10 & 2.7 & \\
\hline \multirow{3}{*}{$\begin{array}{l}\text { Drugs have handling and } \\
\text { disposal instructions }(n= \\
372)\end{array}$} & Yes & 92 & 47.9 & 64 & 35.6 & 156 & 41.9 & \multirow{3}{*}{$\begin{array}{l}\otimes 2= \\
49.180 \\
d f=2 ; \\
P= \\
0.0001^{\star}\end{array}$} \\
\hline & No & 33 & 17.2 & 90 & 50.0 & 123 & 33.1 & \\
\hline & I don't know & 67 & 34.9 & 26 & 14.4 & 93 & 25.0 & \\
\hline \multirow[t]{3}{*}{$\begin{array}{l}\text { Drugs are poison when } \\
\text { abused }(n=372)\end{array}$} & True & 166 & 86.5 & 165 & 91.7 & 331 & 89.0 & \multirow{3}{*}{$\begin{array}{l}\bigotimes 2= \\
2.570 ; \\
d f=1 \\
P=0.109\end{array}$} \\
\hline & False & 26 & 13.5 & 15 & 8.3 & 41 & 11.0 & \\
\hline & & & & & & & & \\
\hline \multirow{4}{*}{$\begin{array}{l}\text { Drugs have expiry date }(n= \\
372)\end{array}$} & Yes & 173 & 90.1 & 175 & 97.2 & 348 & 93.5 & \multirow{3}{*}{$\begin{array}{l}\otimes 2= \\
17.737 \\
d f=2\end{array}$} \\
\hline & No & 6 & 3.1 & 5 & 2.8 & 11 & 3.0 & \\
\hline & I don't know & 13 & 6.8 & 0 & 0.0 & 13 & 3.5 & \\
\hline & & & & & & & & $0.0001^{*++}$ \\
\hline $\begin{array}{l}\text { Key: } * \text { = significant different be } \\
\text { Bqurgogecould be used after }\end{array}$ & $\begin{array}{l}\text { leen low-densi } \\
\text { Yes }\end{array}$ & $\begin{array}{l}\text { and } h i \\
171\end{array}$ & $\begin{array}{l}h-d e n s \\
89.1\end{array}$ & $\begin{array}{l}y \text { sett } \\
160\end{array}$ & $\begin{array}{l}\text { ments, } \\
88.9\end{array}$ & $\begin{array}{l}+=L i \\
331\end{array}$ & $\begin{array}{l}\text { ly rati } \\
89.0\end{array}$ & chi $\mathbb{\nabla 2}=$ \\
\hline
\end{tabular}




\begin{tabular}{|c|c|c|c|c|c|c|c|c|}
\hline \multirow[t]{3}{*}{$\begin{array}{l}\text { expiry without adverse } \\
\text { consequences }(n=372)\end{array}$} & No & 11 & 5.7 & 15 & 8.3 & 26 & 7.0 & \multirow[t]{2}{*}{2.263} \\
\hline & I don't know & 10 & 5.2 & 5 & 2.8 & 15 & 4.0 & \\
\hline & & & & & & & & $\begin{array}{l}d f=2 \\
p=0.323\end{array}$ \\
\hline \multirow{3}{*}{$\begin{array}{l}\text { Unused and expired drugs } \\
\text { could be disposed as solid } \\
\text { waste }(n=372)\end{array}$} & Yes & 103 & 53.6 & 152 & 84.4 & 255 & 68.5 & \multirow{3}{*}{ 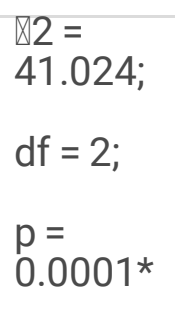 } \\
\hline & No & 37 & 19.3 & 13 & 7.2 & 50 & 13.4 & \\
\hline & I don't know & 52 & 27.1 & 15 & 8.3 & 67 & 18.0 & \\
\hline \multirow{3}{*}{$\begin{array}{l}\text { Drugs disposed with solid } \\
\text { wastes are harmful to the } \\
\text { environment and man }(n= \\
372)\end{array}$} & Yes & 77 & 40.1 & 50 & 27.8 & 127 & 34.1 & \multirow{3}{*}{$\begin{array}{l}\bowtie 2= \\
27.132 ; \\
d f=2 ; \\
p= \\
0.0001 *\end{array}$} \\
\hline & No & 52 & 27.1 & 96 & 53.3 & 148 & 39.8 & \\
\hline & I don't know & 63 & 32.8 & 34 & 18.9 & 97 & 26.1 & \\
\hline \multirow{3}{*}{$\begin{array}{l}\text { There are health } \\
\text { consequences of using } \\
\text { expired drugs ( } n=372)\end{array}$} & Yes & 156 & 81.2 & 136 & 75.6 & 292 & 78.5 & \multirow{3}{*}{$\begin{array}{l}\bowtie 2= \\
37.668 ; \\
d f=2 ; \\
p= \\
0.0001 *\end{array}$} \\
\hline & No & 6 & 3.1 & 37 & 20.6 & 43 & 11.6 & \\
\hline & I don't know & 30 & 15.6 & 7 & 3.9 & 37 & 9.9 & \\
\hline \multirow[t]{2}{*}{ Overall knowledge $(n=371)$} & $\begin{array}{l}\text { Low } \\
\text { knowledge }\end{array}$ & 64 & 33.3 & 109 & 60.9 & 173 & 46.6 & \multirow{2}{*}{$\begin{array}{l}\otimes 2= \\
28.274 ; \\
d f=1 ; \\
p= \\
0.0001 *\end{array}$} \\
\hline & $\begin{array}{l}\text { High } \\
\text { knowledge }\end{array}$ & 128 & 66.7 & 70 & 39.1 & 198 & 53.4 & \\
\hline
\end{tabular}

Also, 47.9\% (LDS) knew that drugs have disposal instructions while 50.0\% (HDS) was not aware in the study area. Drugs are recognised as poison when abused, with $86.5 \%$ and $91.7 \%$ in LDS and HDS while over 9 out of 10 respondents knew that drugs have expiry dates, though $89.1 \%$ and $88.9 \%$ of respondents, respectively in LDS and HDS believed drugs could be used after expiry without adverse consequences. In LDS, $40.1 \%$ of respondents knew when drugs disposed of with solid waste are dangerous to man and his environment, in contrast to those of $53.3 \%$ respondents in HDS. The composite knowledge of respondents presented a high (66.7\%) and low (60.9\%) knowledge base among respondents living in LDS and HDS, respectively and many of the knowledge variables showed a significant difference between LDS and HDS (Table 3).

\section{Attitude on expiry and disposal of expired and unused medication}


Respondents in LDS and HDS believe that households should acquire drugs using doctor's prescription with an overall $89.2 \%$ and at least $87.5 \%$ among respondents in LDS. Though a higher proportion of respondents believed that over the counter (OTC) medications can be acquired without a doctor prescription (51.3\%) with a higher proportion in HDS. Herbal formulations without National agency for food drugs administration control (NAFDAC) No. and those with NAFDAC's No. were higher among residents in HDS, respectively with $67.8 \%$ and $77.2 \%$. moreover, $76.3 \%$ believed customers should request to see the expiry dates on OTC counting drugs before purchase with a higher proportion among LDS (80.7\%) respondents, as well as packaged medications (93.8\%), with a higher percentage among HDS (94.4\%), especially before repeated use of home stored drugs by $80.9 \%$ of respondents (Table 4 ). 
Table 4

Attitude of respondents on management of expired and unused medication

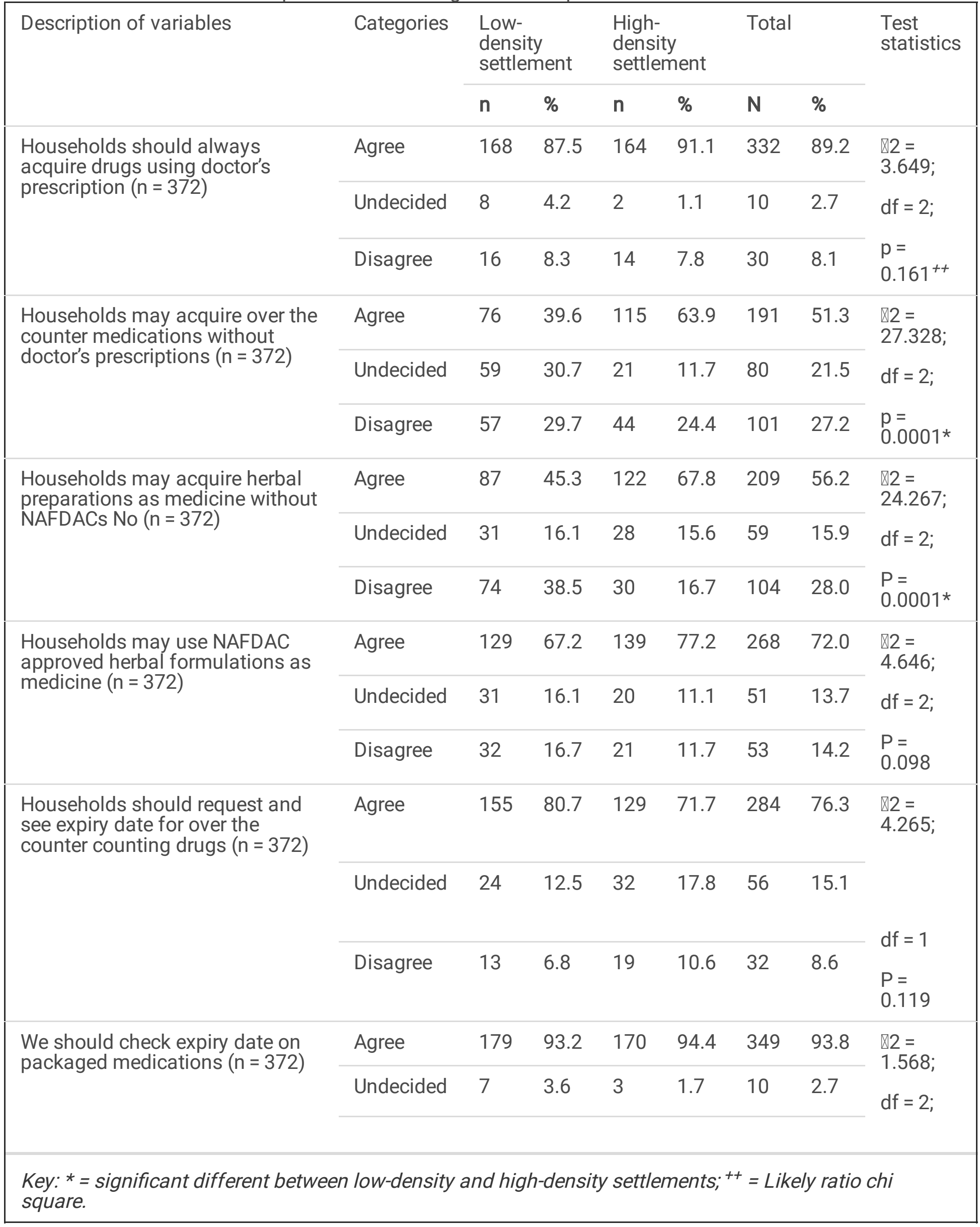




\begin{tabular}{|c|c|c|c|c|c|c|c|c|}
\hline & & \multirow{2}{*}{$\begin{array}{l}p= \\
0.457^{++}\end{array}$} \\
\hline & Disagree & 6 & 3.1 & 7 & 3.9 & 13 & 3.5 & \\
\hline \multirow{3}{*}{$\begin{array}{l}\text { We should check expiry date } \\
\text { before repeated use of stored } \\
\text { drugs }(n=372)\end{array}$} & Agree & 146 & 76.0 & 155 & 86.1 & 301 & 80.9 & \multirow{3}{*}{$\begin{array}{l}\otimes 2= \\
7.656 \\
d f=2 \\
p= \\
0.022 *\end{array}$} \\
\hline & Undecided & 24 & 12.5 & 9 & 5.0 & 33 & 8.9 & \\
\hline & Disagree & 22 & 11.5 & 16 & 8.9 & 38 & 10.2 & \\
\hline \multirow{3}{*}{$\begin{array}{l}\text { One may store unused } \\
\text { medications at home }(n=372)\end{array}$} & Agree & 86 & 44.8 & 126 & 70.0 & 212 & 57.0 & \multirow{3}{*}{$\begin{array}{l}\bowtie 2= \\
30.707 ; \\
d f=2 ; \\
p= \\
0.0001 *\end{array}$} \\
\hline & Undecided & 49 & 25.5 & 13 & 7.2 & 62 & 16.7 & \\
\hline & Disagree & 57 & 29.7 & 41 & 22.8 & 98 & 26.3 & \\
\hline \multirow[t]{3}{*}{$\begin{array}{l}\text { One should dispose of expired } \\
\text { medications }(n=370)\end{array}$} & Agree & 154 & 81.1 & 149 & 82.8 & 303 & 81.9 & \multirow{3}{*}{$\begin{array}{l}\varangle 2= \\
0.194 \\
d f=2 \\
p= \\
0.907 *\end{array}$} \\
\hline & Undecided & 12 & 6.3 & 10 & 5.6 & 22 & 5.9 & \\
\hline & Disagree & 24 & 12.6 & 21 & 11.7 & 45 & 12.2 & \\
\hline \multirow{3}{*}{$\begin{array}{l}\text { One should check for disposal } \\
\text { instructions on all medications ( } \\
=370 \text { ) }\end{array}$} & Agree & 135 & 71.1 & 81 & 45.0 & 216 & 58.4 & \multirow{3}{*}{$\begin{array}{l}\otimes 2= \\
36.245 ; \\
d f=2 ; \\
p= \\
0.0001 *\end{array}$} \\
\hline & Undecided & 41 & 21.6 & 46 & 25.6 & 87 & 23.5 & \\
\hline & Disagree & 14 & 7.4 & 53 & 29.4 & 67 & 18.1 & \\
\hline \multirow{3}{*}{$\begin{array}{l}\text { One should follow disposal } \\
\text { instructions on all medications ( } \mathrm{n} \\
=370 \text { ) }\end{array}$} & Agree & 139 & 73.2 & 83 & 46.1 & 222 & 60.0 & \multirow{3}{*}{$\begin{array}{l}\otimes 2= \\
32.240 ; \\
d f=1 ; \\
p= \\
0.0001 *\end{array}$} \\
\hline & Undecided & 33 & 17.4 & 45 & 25.0 & 78 & 21.1 & \\
\hline & Disagree & 18 & 9.5 & 52 & 28.9 & 70 & 18.9 & \\
\hline \multirow[t]{3}{*}{$\begin{array}{l}\text { One may dispose of all UEMs } \\
\text { with solid waste }(n=371)\end{array}$} & Agree & 93 & 48.7 & 138 & 76.7 & 231 & 62.3 & \multirow{3}{*}{$\begin{array}{l}\bigotimes 2= \\
32.669 \\
d f=1\end{array}$} \\
\hline & Undecided & 62 & 32.5 & 21 & 11.7 & 83 & 22.4 & \\
\hline & Disagree & 36 & 18.8 & 21 & 11.7 & 57 & 15.4 & \\
\hline Overall attitude $(n=372)$ & Positive & 143 & 74.5 & 122 & 67.8 & 265 & 71.2 & $\begin{array}{l}\rrbracket 2= \\
2.036\end{array}$ \\
\hline $\begin{array}{l}\text { Key: * = significant different betwe } \\
\text { square. }\end{array}$ & 1 & d & & & & & & \\
\hline
\end{tabular}




\begin{tabular}{|c|c|c|c|c|c|c|c|}
\hline & & & & & & & \multirow{2}{*}{$\begin{array}{l}d f=1 \\
p=0.154\end{array}$} \\
\hline & 49 & 25.5 & 58 & 32.2 & 107 & 28.8 & \\
\hline
\end{tabular}

A higher proportion of respondents in LDS (70.0\%) believe that drugs could be stored at home and disposed of when expired (81.9\%). Further, homes should check (58.4\%) and follow (60.0\%) disposal instructions on all medications with higher proportions among inmates living in LDS, respectively with $71.1 \%$ and $73.2 \%$. The overall attitude score revealed a not-significant $(p=0.154)$ but a high positive rating in LDS $(74.5 \%)$ and HDS (67.8\%), respectively. Also, a significant difference exists in the proportions of seven attitude indicators out of 12 assessed (Table 4). The assessment of perception showed a significant difference between LDS and HDS $(p=00001)$ respondents. The higher proportion of respondents $(66.7 \%)$ in LDS exhibited a high perception score when compared with those in HDS where a higher proportion (56.1\%) showed a low perception (Fig. 1).

\section{Household strategies for disposing of unused and expired medicines}

Many respondents (71.8\%) are ignorant of any protocol for managing unused and expired medications with a similar trend and a higher proportion among those residents in HDS (76.7\%) when compared with those in LDS (67.2\%). Significant differences exist among residents in LDS and HDS on awareness on the protocol for UEM disposal in Nigeria, though only $24.5 \%$ are aware of any advertorial on the safe management of UEMs with a higher proportion in $\operatorname{LDS}(31,2 \%)$, in most cases through radio (36.3\%), television $(35.3 \%)$ and social media (16.5\%) handles (Table 5). 
Table 5

Household strategies for handling and disposing unused and expired medicines.

\begin{tabular}{|c|c|c|c|c|c|c|c|c|}
\hline \multirow[t]{2}{*}{ Description of variables } & \multirow[t]{2}{*}{ Categories } & \multicolumn{2}{|c|}{$\begin{array}{l}\text { Low- } \\
\text { density } \\
\text { settlement }\end{array}$} & \multicolumn{2}{|c|}{$\begin{array}{l}\text { High- } \\
\text { density } \\
\text { settlement }\end{array}$} & \multicolumn{2}{|c|}{ Total } & \multirow[t]{2}{*}{$\begin{array}{l}\text { Test } \\
\text { statistics }\end{array}$} \\
\hline & & $\mathrm{n}$ & $\%$ & $\mathrm{n}$ & $\%$ & $\mathbf{N}$ & $\%$ & \\
\hline \multirow{3}{*}{$\begin{array}{l}\text { Awareness of protocol } \\
\text { for managing UEMs in } \\
\text { Nigeria }(n=372)\end{array}$} & Yes & 37 & 19.3 & 23 & 12.8 & 60 & 16.1 & \multirow{3}{*}{$\begin{array}{l}\searrow 2= \\
4.276 ; \\
d f=2 ; \\
p= \\
0.118^{\star}\end{array}$} \\
\hline & No & 129 & 67.2 & 138 & 76.7 & 267 & 71.8 & \\
\hline & I don't know & 26 & 13.5 & 19 & 10.6 & 45 & 12.1 & \\
\hline \multirow{3}{*}{$\begin{array}{l}\text { Are there advertorial on } \\
\text { management of UEMs } \\
(n=372)\end{array}$} & Yes & 60 & 31.2 & 31 & 17.2 & 91 & 24.5 & \multirow{3}{*}{$\begin{array}{l}\bigotimes 2= \\
37.309 \\
d f=2 ; \\
p= \\
0.0001 *\end{array}$} \\
\hline & No & 73 & 38.0 & 125 & 69.4 & 198 & 53.2 & \\
\hline & I don't know & 59 & 30.7 & 24 & 13.3 & 83 & 22.3 & \\
\hline \multirow{5}{*}{$\begin{array}{l}\text { Awareness channels on } \\
\text { the management of } \\
\text { UEMs }(n=91)\end{array}$} & Radio & 22 & 36.5 & 11 & 35.5 & 33 & 36.3 & \multirow{5}{*}{$\begin{array}{l}\otimes 2= \\
4.898 \\
d f=4 \\
p= \\
0.298^{++}\end{array}$} \\
\hline & TV & 21 & 35.0 & 11 & 35.5 & 32 & 35.3 & \\
\hline & Newspaper & 4 & 6.7 & 0 & 0.9 & 4 & 4.4 & \\
\hline & Social media & 10 & 16.7 & 5 & 16.1 & 15 & 16.5 & \\
\hline & Electronic media & 3 & 5.0 & 4 & 12.9 & 7 & 7.7 & \\
\hline \multirow{3}{*}{$\begin{array}{l}\text { Management of UEMs } \\
\text { post illness recovery }(n= \\
372)\end{array}$} & Keep them & 58 & 30.2 & 106 & 58.9 & 164 & 44.1 & \multirow{3}{*}{$\begin{array}{l}\otimes 2= \\
33.182 ; \\
d f=2 ; \\
p= \\
0.0001^{\star}\end{array}$} \\
\hline & $\begin{array}{l}\text { Give to people } \\
\text { with similar } \\
\text { illness }\end{array}$ & 24 & 12.5 & 20 & 11.1 & 44 & 11.8 & \\
\hline & $\begin{array}{l}\text { Discard with } \\
\text { waste }\end{array}$ & 110 & 57.3 & 54 & 30.0 & 164 & 44.1 & \\
\hline \multirow{5}{*}{$\begin{array}{l}\text { How households } \\
\text { manage 'solid' UEM ( } n= \\
372)\end{array}$} & $\begin{array}{l}\text { Give to other } \\
\text { people }\end{array}$ & 23 & 12.0 & 9 & 5.0 & 32 & 8.6 & \multirow{5}{*}{$\begin{array}{l}\bigotimes 2= \\
20.584 ; \\
d f=4 ; \\
p= \\
0.0001^{\star}\end{array}$} \\
\hline & $\begin{array}{l}\text { Disposed in } \\
\text { dustbin with } \\
\text { waste }\end{array}$ & 140 & 72.9 & 122 & 67.8 & 262 & 70.4 & \\
\hline & $\begin{array}{l}\text { Pour in the } \\
\text { toilet/sink }\end{array}$ & 10 & 5.2 & 6 & 3.3 & 16 & 4.3 & \\
\hline & Bury them & 5 & 2.6 & 4 & 2.2 & 9 & 2.4 & \\
\hline & Burn them & 14 & 7.3 & 39 & 21.7 & 53 & 14.2 & \\
\hline
\end{tabular}

Key: ${ }^{*}=$ significant different between low-density and high-density settlements; ${ }^{++}=$Likely ratio hi square. 


\begin{tabular}{|c|c|c|c|c|c|c|c|c|}
\hline \multirow{5}{*}{$\begin{array}{l}\text { How households } \\
\text { manage 'liquid' UEM ( } n= \\
372)\end{array}$} & $\begin{array}{l}\text { Give to other } \\
\text { people }\end{array}$ & 18 & 9.4 & 4 & 2.2 & 22 & 5.9 & \multirow{5}{*}{$\begin{array}{l}\otimes 2= \\
50.338 \\
d f=4 ; \\
p= \\
0.0001 \star\end{array}$} \\
\hline & $\begin{array}{l}\text { Disposed in } \\
\text { dustbin with } \\
\text { waste }\end{array}$ & 92 & 47.9 & 100 & 55.6 & 192 & 51.6 & \\
\hline & $\begin{array}{l}\text { Pour in the } \\
\text { toilet/sink }\end{array}$ & 69 & 35.9 & 31 & 17.2 & 100 & 26.9 & \\
\hline & Bury them & 7 & 3.6 & 7 & 3.9 & 14 & 3.8 & \\
\hline & Burn them & 6 & 3.1 & 38 & 21.1 & 44 & 11.8 & \\
\hline \multirow{6}{*}{$\begin{array}{l}\text { Type of solid waste } \\
\text { storage receptacle }(n= \\
372)\end{array}$} & Nylon/Cellophane & 41 & 21.4 & 27 & 15.0 & 68 & 18.3 & \multirow{6}{*}{$\begin{array}{l}\otimes 2= \\
17.305 ; \\
d f=5 ; \\
p= \\
0.004^{\star++}\end{array}$} \\
\hline & Bucket with lid & 30 & 15.6 & 15 & 8.3 & 45 & 12.1 & \\
\hline & Basket & 37 & 19.3 & 66 & 36.7 & 103 & 27.7 & \\
\hline & Carton & 22 & 11.5 & 17 & 9.4 & 39 & 10.5 & \\
\hline & Standard bin & 60 & 31.2 & 52 & 28.9 & 112 & 30.1 & \\
\hline & Others & 2 & 1.0 & 3 & 1.7 & 5 & 1.3 & \\
\hline \multirow[t]{2}{*}{$\begin{array}{l}\text { Solid waste receptacle } \\
\text { has a cover }(n=372)\end{array}$} & Yes & 122 & 63.5 & 105 & 58.3 & 227 & 61.0 & \multirow{2}{*}{$\begin{array}{l}\otimes 2= \\
1.060 ; \\
d f=2 ; \\
p=0.303\end{array}$} \\
\hline & No & 70 & 36.5 & 75 & 41.7 & 145 & 39.0 & \\
\hline \multirow[t]{5}{*}{$\begin{array}{l}\text { Solid waste disposal } \\
\text { strategy }(n=372)\end{array}$} & $\begin{array}{l}\text { Pick up by refuse } \\
\text { truck }\end{array}$ & 71 & 37.0 & 69 & 38.3 & 140 & 37.6 & \multirow{5}{*}{$\begin{array}{l}\otimes 2= \\
62.102 \\
d f=4 ; \\
p= \\
0.0001^{*++}\end{array}$} \\
\hline & $\begin{array}{l}\text { Dump in any } \\
\text { nearby bush }\end{array}$ & 36 & 18.8 & 11 & 6.1 & 47 & 12.6 & \\
\hline & $\begin{array}{l}\text { Carry to a } \\
\text { communal bin }\end{array}$ & 39 & 20.3 & 6 & 3.3 & 45 & 12.1 & \\
\hline & $\begin{array}{l}\text { Burn in the } \\
\text { compound }\end{array}$ & 40 & 20.8 & 91 & 50.6 & 131 & 35.2 & \\
\hline & Bury in a pit & 6 & 3.1 & 3 & 1.7 & 9 & 2.4 & \\
\hline \multirow[t]{3}{*}{$\begin{array}{l}\text { Who routinely dispose of } \\
\text { waste? }\end{array}$} & $\begin{array}{l}\text { Children }(\text { aged < } \\
10 \text { years) }\end{array}$ & 1 & 0.5 & 0 & 0.0 & 1 & 0.3 & \multirow{3}{*}{$\begin{array}{l}\otimes 2= \\
10.074 ; \\
d f=2 ; \\
p= \\
0.006^{\star++}\end{array}$} \\
\hline & $\begin{array}{l}\text { Adolescents } \\
\text { (aged 10- } \\
19 \text { years) }\end{array}$ & 81 & 42.2 & 50 & 27.8 & 131 & 35.2 & \\
\hline & $\begin{array}{l}\text { Adults (aged } \geq \\
20 \text { years) }\end{array}$ & 110 & 57.3 & 130 & 72.2 & 240 & 64.5 & \\
\hline
\end{tabular}


The study showed that households disposed of 'solid' and 'liquid' UEMs in dust bin with $70.4 \% 51.6 \%$, respectively. For 'solid' UEMs, households living in LDS reported a higher proportion (72.9\%) while for 'liquid' UEMs, those in HDS reported a higher proportion with $55.6 \%$. Disposal of solid and liquid UEMs showed a significant difference in practice between LDS and HDS. However, solid waste is mostly disposed of by refuse truck (37.6\%), by burning (35.2\%) and dumping in nearby bush open dumps (12.6\%). Disposal through refuse trucks and burning in the compound predominates among households in HDS while dumping in nearby bush/open dumps is mostly practised by residents in LDS (Table 5).

\section{Perceived consequences of indiscriminate disposal of unused and expired medicines}

As shown in Fig. 2, 37.1\% of respondents, stratified into LDS (52.6\%) and HDS (20.6\%) perceived there are consequences associated with discarded unused and expired medicines, with a significant difference in responses between LDS and HDS responses $(p=0001)$. The perceived consequences mostly mentioned by respondents in LDS and HDS, respectively include accidental ingestion (76.6\% vs $26.7 \%$ ) and land pollution (69.6\% vs $36.7 \%)$ and water pollution ( $72.4 \%$ vs $32.8 \%$ ) and drugs toxicity ( $58.3 \%$ vs $32.8 \%)$, in LDS and HDS.

\section{The abundance of unused and expired medicines in the study area}

Table 6 presents the abundance of unused and expired medication in the homes of respondents. $41.4 \%$, $31.5 \%, 33.1 \%, 61.6 \%$ and $79.8 \%$ of households do not have antibiotics, antimalaria, analgesics, cough, and anti-diarrhoea drugs in their homes at the time of the survey. However, $49.7 \%, 57.3 \%, 52.7 \%, 32.5 \%$ and $12.9 \%$ had 1-2 sachets of antibiotics, antimalaria, analgesics, cough, and anti-diarrhoea drugs in their homes at the time when surveyed. Besides, there was a significant difference in the drugs abundance between LDS and HDS $(p=0.0001)$, with LDS having the higher abundance in their homes for antibiotics, antimalaria and analgesics and cough-related drugs while those in HDS have a higher abundance of anti-diarrhoea medicines. 
Table 6

Abundance of unused and expired medicines in respondent's homes.

\begin{tabular}{|c|c|c|c|c|c|c|c|c|}
\hline \multirow[t]{2}{*}{ Variables } & \multirow[t]{2}{*}{ Categories } & \multicolumn{2}{|l|}{ LDS } & \multicolumn{2}{|l|}{ HDS } & \multicolumn{2}{|c|}{ Total } & \multirow{2}{*}{$\begin{array}{l}\text { Test } \\
\text { statistics }\end{array}$} \\
\hline & & $\mathbf{n}$ & $\%$ & $\mathbf{n}$ & $\%$ & $\mathbf{N}$ & $\%$ & \\
\hline \multirow[t]{3}{*}{ Antibiotics ( $n=372)$} & None & 68 & 35.4 & 86 & 47.8 & 154 & 41.4 & \multirow{3}{*}{$\begin{array}{l}\bowtie 2= \\
27.235 \\
d f=2 ; \\
p= \\
0.0001 *\end{array}$} \\
\hline & $\begin{array}{l}1-2 \\
\text { sachets }\end{array}$ & 93 & 48.4 & 92 & 51.1 & 185 & 49.7 & \\
\hline & $\begin{array}{l}\geq 3 \\
\text { sachets }\end{array}$ & 31 & 16.1 & 2 & 1.1 & 33 & 8.9 & \\
\hline \multirow[t]{3}{*}{ Antimalaria $(n=372)$} & None & 40 & 20.8 & 77 & 42.8 & 117 & 31.5 & \multirow{3}{*}{$\begin{array}{l}\bigotimes 2= \\
48.920 ; \\
d f=2 ; \\
p= \\
0.0001 *\end{array}$} \\
\hline & $\begin{array}{l}1-2 \\
\text { sachets }\end{array}$ & 113 & 58.9 & 100 & 55.6 & 213 & 57.3 & \\
\hline & $\begin{array}{l}\geq 3 \\
\text { sachets }\end{array}$ & 39 & 20.3 & 3 & 1.7 & 42 & 11.3 & \\
\hline \multirow[t]{3}{*}{ Analgesics $(n=372)$} & None & 53 & 27.6 & 70 & 38.9 & 123 & 33.1 & \multirow{3}{*}{$\begin{array}{l}\bowtie 2= \\
67.997 ; \\
d f=2 ; \\
p= \\
0.0001 *\end{array}$} \\
\hline & $\begin{array}{l}1-2 \\
\text { sachets }\end{array}$ & 87 & 45.3 & 109 & 60.6 & 196 & 52.7 & \\
\hline & $\begin{array}{l}\geq 3 \\
\text { sachets }\end{array}$ & 52 & 27.1 & 1 & 0.6 & 53 & 14.2 & \\
\hline \multirow{3}{*}{$\begin{array}{l}\text { Cough and cough related }(n= \\
372)\end{array}$} & None & 112 & 58.3 & 117 & 65.0 & 229 & 61.6 & \multirow{3}{*}{$\begin{array}{l}\bowtie 2= \\
16.825 ; \\
d f=2 ; \\
p= \\
0.0001 *\end{array}$} \\
\hline & $\begin{array}{l}1-2 \\
\text { sachets }\end{array}$ & 60 & 31.2 & 61 & 33.9 & 121 & 32.5 & \\
\hline & $\begin{array}{l}\geq 3 \\
\text { sachets }\end{array}$ & 20 & 10.4 & 2 & 1.1 & 22 & 5.9 & \\
\hline \multirow[t]{2}{*}{ Anti-diarrhoea $(n=372)$} & None & 148 & 77.1 & 149 & 82.8 & 297 & 79.8 & \multirow{2}{*}{$\begin{array}{l}\otimes 2= \\
41.189 ; \\
d f=2 ; \\
p= \\
0.0001 *\end{array}$} \\
\hline & $\begin{array}{l}1-2 \\
\text { sachets }\end{array}$ & 17 & 8.9 & 31 & 17.2 & 48 & 12.9 & \\
\hline
\end{tabular}

\section{Predictors of unused and expired medicines}

The binary logistic regression predicting awareness on disposal protocol revealed that 'marital status' (0.008), 'single parenthood' (0.011) and 'other' 'marital status' (0.031) subsets, 'occupation' (0.031) and 'civil servant' 
(0.024) subset and 'high knowledge' (0.0001) are predictors of awareness of UEM disposal guidelines are significant predictors $(P<0.05)($ Table 7$)$. 
Table 7

Binary logistic regression on awareness of UEM disposal protocol

\begin{tabular}{|c|c|c|c|c|c|}
\hline \multirow[t]{2}{*}{ Variables } & \multirow[t]{2}{*}{ B-coefficient } & \multirow[t]{2}{*}{ Odd Ratio } & \multicolumn{2}{|l|}{$95 \% \mathrm{Cl}$} & \multirow[t]{2}{*}{$P$ value } \\
\hline & & & Lower & Upper & \\
\hline Marital status & & & & & $0.008^{\star}$ \\
\hline \multicolumn{6}{|l|}{ Single (Ref) } \\
\hline Single parenthood & -3.162 & 0.042 & 0.004 & 0.480 & $0.011^{*}$ \\
\hline Married & -0.162 & 0.851 & 0.185 & 3.911 & 0.836 \\
\hline Others (widowed, divorced, separated) & -1.373 & 0.253 & 0.072 & 0.885 & $0.031^{*}$ \\
\hline Toilet type & & & & & 0.630 \\
\hline \multicolumn{6}{|l|}{ Pit latrine (Ref) } \\
\hline None & -0.394 & 0.674 & 0.257 & 1.765 & 0.422 \\
\hline Water closet & 0.319 & 1.376 & 0.220 & 8.590 & 0.733 \\
\hline Wealth categories & & & & & 0.293 \\
\hline \multicolumn{6}{|l|}{ Poor (Ref) } \\
\hline Average & -0.872 & 0.418 & 0.137 & 1.278 & 0.126 \\
\hline Rich & -0.646 & 0.524 & 0.195 & 1.408 & 0.200 \\
\hline Age categories (years) & & & & & 0.953 \\
\hline \multicolumn{6}{|l|}{ 18-24 (Ref) } \\
\hline $25-44$ & -0.125 & 0.882 & 0.126 & 6.169 & 0.900 \\
\hline$\geq 45$ & 0.100 & 1.105 & 0.451 & 2.708 & 0.827 \\
\hline Occupation & & & & & $0.031^{*}$ \\
\hline \multicolumn{6}{|l|}{ Self-employed (Ref) } \\
\hline civil servant & -0.939 & 0.391 & 0.173 & 0.886 & $0.024^{\star}$ \\
\hline Others (full-housewife, unemployed) & 0.268 & 1.308 & 0.485 & 3.523 & 0.596 \\
\hline private sector employed & -0.438 & 0.645 & 0.181 & 2.306 & 0.500 \\
\hline \multicolumn{6}{|l|}{ Attitude } \\
\hline Negative (Ref) & & & & & \\
\hline
\end{tabular}

Key: Ref = Variable used for Reference; LDS = low density settlement; HDS = high density settlement; ${ }^{*}=p$ $<0.05$. 


\begin{tabular}{|c|c|c|c|c|c|}
\hline \multirow[t]{2}{*}{ Variables } & \multirow[t]{2}{*}{ B-coefficient } & \multirow[t]{2}{*}{ Odd Ratio } & \multicolumn{2}{|c|}{$95 \% \mathrm{Cl}$} & \multirow[t]{2}{*}{$P$ value } \\
\hline & & & Lower & Upper & \\
\hline Positive & 0.376 & 1.456 & 0.457 & 4.643 & 0.525 \\
\hline \multicolumn{6}{|c|}{ Perception } \\
\hline \multicolumn{6}{|l|}{ Low (Ref) } \\
\hline High & -0.179 & 0.836 & 0.245 & 2.853 & 0.775 \\
\hline \multicolumn{6}{|c|}{ Knowledge } \\
\hline \multicolumn{6}{|l|}{ Low (Ref) } \\
\hline High & -1.673 & 0.188 & 0.075 & 0.470 & $0.0001 *$ \\
\hline \multicolumn{6}{|c|}{ Settlement density } \\
\hline \multicolumn{6}{|l|}{ LDS (Ref) } \\
\hline HDS & 0.181 & 1.199 & 0.557 & 2.578 & 0.643 \\
\hline Constant & 0.832 & 2.299 & & & 0.405 \\
\hline \multicolumn{6}{|c|}{$\begin{array}{l}\text { Key: Ref = Variable used for Reference; LDS = low density settlement; HDS = high density settlement; }{ }^{*}=p \\
<0.05 .\end{array}$} \\
\hline
\end{tabular}

\section{Discussion}

The paucity of guidelines for household management of unused and expired medications is being discussed in Nigeria and across the world. Current research endeavour is focussing on the emergence of sound disposal processes, which is a mirage in many developing countries. In Nigeria, medicines and other regulated products are labelled as 'unwholesome' when expired, improperly sealed and leaking, damaged, improperly stored, improperly labelled, counterfeit, substandard and adulterated, prohibited and unauthorized [22]. This made Asa [23] in 2011 and Michael et al. [24] in 2019 to assess the prevailing practices, respectively among households and community pharmacists in the disposal of expired drugs in Lagos and Anambra states in Nigeria. However, the national guidelines for the destruction of medicines and other regulated products were tailored for institutional-based consignments, while there was no provision or guide for management of UEMs from households [22]. Hence, the current study examined the abundance, household disposal practices and consequences of current management regimen for unused and expired medicines in the study area.

\section{Socio-demographic characteristics}

The current study purposively selected females in randomly selected households as respondents, who are the custodian of medicines, according to recognizance information in households, in contrast to Udofia et al. in Ghana where male and female community respondents were studied [9]; in Kabul, where most respondents 
were male and female university graduates [7], in Turkey, where more women reportedly kept unused drugs at home when compared to men [25], and in Nigeria, where the respondents were community-based pharmacists and involved both sexes [24].

In our study, most respondents were mid-adults in agreement with the respondents' age in the Kabul study [7] while most respondents were Christians, also in congruence with the study in Adigrat city, northern Ethiopia [26]. This is a productive population that is active in childbearing and innately tasked with caring for the wellbeing of the family. In this regard, a majority of respondents are married and had at least a secondary education in consonance with the findings in Kabul, where $38.6 \%$ respondents had up to secondary education and $54.2 \%$ were university graduates [7]. Most respondents are married in LDS and HDS, though higher than those reported in Ethiopia (52.2\%) [27] and more respondents had and use water closet toilet in the LDS (76.0\%) than in the HDS (47.2\%) settlements and can discard of liquid UEMs easily in their abode. Also, there were significant differences in all socio-demographic characteristics of respondents, between residents in LDS and HDS, indicating a disparity between the study groups.

\section{Drugs acquisition and duration of use characteristics}

Most respondents in LDS and HDS received drugs as part of treatment, though the inventory of drugs in respondents' homes was predominantly acquired through self-medication, for treatment of malaria and diarrhoea related ailments. This may be responsible for the observations by Barnett-Itzhaki et al. that large amounts of expired and unused medications accumulate in households [4]. It can, therefore, be hypothesized that people use, and complied with, a drug regimen, in most cases when prescribed as part of treatment by healthcare professionals with the tendency of those acquired through self-medication largely responsible for UEMs at the household level, as observed in Indonesia [28]. The abundance of UEMs in homes was due to non-compliance (56.8\%) with the prescribed drug use regimen, due to feeling of recovery and the emergence of side effects [28-30]. This observation was consistent with the study findings in Ethiopia where mostly due to recovery/disappearance of disease symptoms in most cases [27].

\section{Knowledge of expiry and disposal of expired and unused medication}

For drugs, compliance with medicines safety protocol is germane to keep the active pharmaceutical ingredients (API) active within their shelf life. Some medicines are to be kept active at a threshold of weather conditions. However, many households and users are ignorant of some stringent conditions to keep the API active. According to Laitmeyer and Adhoch, the United States Food and Drug Administration's (USFDA) defined expiry date (designated by the manufacturers) is "the date placed on the container/labels of an API designating the time during which the API is expected to remain within established shelf-life specifications if stored under defined conditions and after which it should not be used" [31]. This has become worse with climate change, with environmental conditions exceeding the usual cold and warm temperatures, which also is expected to influence the API. Besides, knowledge has been mooted as influencing attitude and practice, though previous studies posited that good knowledge and positive attitude may not translate to good behaviour [15, 32]. 
Most respondents are aware of medicines use instructions by weight and age prescriptions and knew that drugs are poisons and should be handled with care across the two study domains, as observed by Ruhoy and Daughton [34] and Verlicchi et al. [35] study findings. Most drugs sachets lack disposal instructions, a limitation to acquiring sound disposal strategies and this did not stop respondents from recognising drugs as poison when either abused or ignorantly disposed of. Despite most respondents knowing that they have expiry dates, they believed that they can be used after expiry without adverse consequences while a few knew disposing of UEMs with wastes portends danger to human and the environment.

Most respondents in LDS have good knowledge in contrast to those in HDS where a majority has poor knowledge. This is rare in literature and contrast to previous studies [26, 27, 36, 37]. This herald the significant differences in knowledge between respondents in HDS and LDS. Besides, most respondents in the current study demonstrated good knowledge, in agreement with previous studies from Ethiopia, New Zealand and Turkey [26, 27, 37, 38], especially respondents in LDS in contrast to those in HDS where a majority has poor knowledge, in congruence with a study in Kenya [39], perhaps due to diversity in their socio-demographic characteristics. In the study area, sound UEMs management practice goes beyond the capacity of the households due to absence of necessary supportive mechanism and giving people information does not make them change, in any case.

\section{Attitude on expiry and disposal of expired and unused medication}

The general protocol holds among the respondents that medicines should be acquired based on doctors' prescription, as evident in the study. Besides, however, self-medication, through the purchase of medicines over the counter was also widely reported, especially among people in HDS. This situation was deepened since anyone can acquire medicines, without doctor's prescription in the study area, due to laxity in control and enforcement of extant national laws, perhaps to the about 90-220 minutes of wait-time associated with hospital visits by the sick for consultations, examinations and medicines acquisitions [40-44], apart from the non-availability of several essential medicines [45], expected to be provided by the National health insurance scheme in accredited healthcare facilities in Nigeria [46]. These might also be the reason for the increasing prevalence of NAFDAC licenced and unapproved herbal formulations.

Medicines expiry is the date provided on them that guarantees their full potency and safety under good manufacturing practices and several of them, such as vaccine vials, insulin and nitro-glycerin degenerate on expiry $[47,48]$. Our study revealed that many households confirmed medicines expiry on prescription and OTC drugs, as previously reported $[7,9,25,28]$. This could be attributed to the awareness created over the years on the need to ensure that medicines are still within the expiry dates before use. However, the expected medicines keeping conditions may be compromised in many patent medicine outlets, due to poor compliance and enforcement by responsible agencies. In agreement with previous studies, households keep medicines home $[7,25]$. and believe that such should be disposed of when expired. This is mostly supported for selfmedication in terms of emergency sickness and medication uses. The respondents had a positive attitude and high perception ratings, irrespective of the settlement area. This also agrees with findings in Brazil [49] and Ethiopia [26, 27]. 


\section{The abundance of unused and expired medicines in the study area}

Between $31.5 \%$ and $79.8 \%$, households do not keep medicines in homes, though there were differences among households in LDS and HDS, in agreement with studies by Kahsay et al. and Insani et al. where $47.6 \%$ and $4.5 \%$ of respondents do not keep medicines at home [26, 28]. Also, antimalarial, analgesics and antibiotics were predominantly stored by households. The high burden of drugs in homes could only promote inappropriate self-medication in agreement with the position of Asa et al. [23]and drug use without medical advice. The types of drugs in homes in the current study differ slightly when compared with those mentioned in a previous study where Asa et al. in Nigeria, Ayele and Mamu [27] and Kahsay et al. [26] in Ethiopia where analgesics and antibiotics were highest in homes. The changing climate and illnesses endemicity could explain drugs abundance dynamics [23] and Nigeria tropical climate, with malaria being endemic could explain the abundance of antimalaria drugs in homes $[50,51]$. The availability of medicines in households might be due to a lack of a formal system for drug take-back policy and system which portends disposal challenges, especially when expired [52].

\section{Strategy for disposing of unused and expired medicines by households}

A few respondents have seen advertorials on the safe management of damaged and expired medicines through the news and electronic media, indicting a poor public awareness on disposal guidelines for unused, damaged and expired medicines [26, 27, 53]. In another study, respondents attested to paucity of information on disposal of UEMs $[28,54]$. Just as the management of healthcare wastes are not regulated in many countries at the household level [55], there is no protocol either by NAFDAC or NESREA to guide home management of damaged and expired medications in Nigeria, and thus, contributing to the ignorance of households in LDS and HDSs in the study area. These findings agree with previous studies in Australia [28], Nigeria [23] and in systematic reviews which reported similar findings in the USA, New Zealand, Bangladesh, Malta and Ireland [37, 53], with greater consequences in countries with poor solid waste management systems were reported. These studies confirmed the 'absence of a global comprehensive and binding approach', that should encourage a safe medication disposal strategy $[4,28]$. The above position, however, contrasted with that of European Union, where EC 2004 legislation, through Directive 2001/83/EC, Directive 2004/27/EC), Article 127b, direct Member States to establish appropriate collection systems for medicinal products that are unused or expired [37].

In Nigeria, the national environmental standards and regulatory enforcement agency (NESREA) is promoting the 'extended producer responsibility' (EPR) policy [57]. It is a framework for partnership between government, business, and the society for zero waste, through the life cycle approach for medicines from production to safe disposal as a shared responsibility.

Notwithstanding, households in the study area dispose of damaged, unused, and expired medications, either solid or liquid through disposal in dustbins with solid waste, though there were significant disparities in the method used between LDS and HDS. They were disposed of through refuse truck and open burning and open dumping in congruence with those in many studies $[27,29,54,56]$. These disposal methods were used for 
managing solid waste in the study area, however with the danger of APls releases into the environmental media, while toxic pollutants can be released into the air by burning [56], which calls for a separation of UEMs from other households solid waste [54], before management. The study findings align with those in systematic reviews ( $[37,53]$ in over 30 countries, including Kuwait, United Kingdom, Lithuania, Qatar, Serbia, Ghana, Bangladesh, Malta and Saudi Arabia, among others.

\section{Perceived consequences of indiscriminate disposal of unused and expired medicines}

The cradle-to-grave responsibility for medicines and their impact on the global environment is gaining attention [55], especially due to the recognised and perceived environmental and public health consequences associated with the disposal of UEMs. This made some respondents to respectively considered accidental ingestion of leftover and expired medicines, land and water pollution and drugs toxicity, though there was a significant difference between LDS and HDS $(p=0001)$. The reported consequences were in agreement with previous studies in Nigeria [24, 56] and Ethiopia [27] and Malaysia [54], and in systematic reviews [52]. There were trace concentrations of medicine APIs in conventional drinking water resources and landfills leachates [58]. Other consequences include accidental medicine poisonings, diversion/repackaging of UEMs for illicit use and antibiotic resistance, all of which impose socio-economic, human health and ethical burdens at the population level[53]. This was, however, at variance with ignorance reported in Indonesia by $53.1 \%$ of households that unsafe medication disposal practices could harm the environment and population health [28], and are rarely removed in water and wastewater treatment plants [4].

The awareness of respondents on the disposal of UEMs was strongly predicted by marital status, occupation, and knowledge. However, this outcome needs to be interpreted with caution, since the weighting was not applied to detect the true effects of these covariates and on awareness of respondents on the disposal of used and expired medications.

\section{Conclusions}

There was unwholesome disposal of UEMs between residents with solid wastes in low-density and highdensity settlements. Respondents good knowledge and positive attitudes contrasted to poor UEMs disposal practice. Most of the drugs in households were antimalaria and analgesics with solid and liquid medicines disposed of in most cases with solid wastes. The perceived consequences of the current disposal practice include accidental ingestion, land and water pollution and drugs toxicity. Therefore, appropriate legislation should herald sound, and incentive-driven, UEMs return for disposal drop-off systems to designated premises. This should be aided by compliance assessment to drive its recovery and alleviate the negative consequences of current disposal practices in Nigeria.

\section{Abbreviations}

APIs - Active pharmaceutical ingredients

EPR - Extended Producer Responsibility 
HCW - HealthCare Waste

HDS - High-Density Settlement

IBM - International Business Machine

IPH - Institute of Public Health

IQR - Interquartile Range

LDS - Low-Density Settlement

LGA - Local Government Area

NAFDAC National Agency for Food Drugs Administration Control

NESREA National Environmental Standards and Enforcement Regulatory Agency

NCDs - Non-Communicable Diseases

OTC - Over the Counter

SPSS - Statistical Package for Social Sciences

UEM - Unused and Expired Medicines

USFDA - United States Food and Drug Administration's

WHO - World Health Organisation

\section{Declarations}

\section{Ethics approval and consent to participate}

The research and ethics committee of the Institute of Public Health of the Obafemi Awolowo University in Nigeria approved the study protocol (IPH/OAU/12/1008) before to field data collection. The randomly selected households permitted the study while selected respondents gave verbal consent since the study did not involve any invasive procedures [59].

\section{Consent for publication}

Not applicable

\section{Availability of data and materials}

The datasets used and/or analysed during the current study are available from the corresponding author on reasonable request 


\section{Competing interests}

The authors declare that they have no competing interests

\section{Funding}

This research did not receive any specific grant from funding agencies in the public, commercial, or not-forprofit sectors.

\section{Authors' contributions}

GTI, COJ, OTA and OOA conceived the study and all authors contributed to the study design, COJ, GTI, OVO and TEF supervised field data collection, entry, analyses, development, and interpretation of the summary tables. COJ and GTI wrote the introduction and methods. OOA, OVO, OTA and TEF wrote and reviewed the results and discussion while OOA coordinated the integration of feedback into the manuscript draft. All authors contributed to the abstract write-up, vetted, and approved the final draft for submission to the Journal.

\section{Acknowledgements}

The authors acknowledged the immeasurable support of the Water, Sanitation and Hygiene department, the community development association, and households in Ife Central LGA for entry, guidance and field data collection support and cooperation during the field data collection. We appreciate the time and patience of the respondents whose efforts led to data presented in this manuscript.

\section{References}

1. WHO. WHO. Challenges in expanding access to essential medicines. 2007.

2. Katzung. Trevor's. Pharmacology Examination \& Board Review. 2015.

3. Agoston S, Vandenbrom R, Wierda JM. Clinical pharmacokinetics of neuromuscular blocking drugs. Clin Pharmacokinet. 1992;22:94-115. doi:10.2165/00003088-199222020-00002.

4. Barnett-Itzhaki Z, Berman T, Grotto I, Schwartzberg E. Household medical waste disposal policy in Israel. Isr J Health Policy Res. 2016;5:1-8. doi:10.1186/s13584-016-0108-1.

5. Wheeler AJ, Spinks J, Bettington E, Kelly F. Evaluation of the National Return of unwanted medicines (RUM) program in Australia: a study protocol. J Pharm Policy Pract. 2017;10:38. doi:10.1186/s40545017-0126-6.

6. World Health Organization. The World Medicines Situation. WHO. 2004;:1-151.

7. Bashaar M, Thawani V, Hassali MA, Saleem F. Disposal practices of unused and expired pharmaceuticals among general public in Kabul. BMC Public Health. 2017;17:1-8.

8. Bean TG, Bergstrom E, Thomas-Oates J, Wolff A, Bartl P, Eaton B, et al. Evaluation of a Novel Approach for Reducing Emissions of Pharmaceuticals to the Environment. Environ Manage. 2016;58:707-20.

9. Udofia EA, Gulis G, Fobil J. Solid medical waste: a cross-sectional study of household disposal practices and reported harm in Southern Ghana. BMC Public Health. 2017;17:1-12. 
10. Seehusen DA, Edwards J. Patient practices and beliefs concerning disposal of medications. J Am Board Fam Med. 2006;19:542-7.

11. Braund R, Peake BM, Shieffelbien L. Disposal practices for unused medications in New Zealand. Environ Int. 2009;35:952-5.

12. Vogler S, Leopold C, Zuidberg C, Habl C. Medicines discarded in household garbage: Analysis of a pharmaceutical waste sample in Vienna. J Pharm Policy Pract. 2014;7:1-8.

13. WHO (World Health Organization). A global overview of national regulations and standards for drinkingwater quality. 2018. http://apps.who.int/iris/bitstream/handle/10665/272345/9789241513760-eng.pdf? sequence=1\&isAllowed=y. Accessed 21 Jun 2018.

14. Amster ED. Mitigating pharmaceutical waste exposures: Policy and program considerations. Isr J Health Policy Res. 2016;5:1-4. doi:10.1186/s13584-016-0118-z.

15. Kusturica MP, Sabo A, Tomic Z, Horvat O, Šolak Z. Storage and disposal of unused medications: Knowledge, behavior, and attitudes among Serbian people. Int J Clin Pharm. 2012;34:604-10.

16. Higgins CP, Paesani ZJ, Chalew TEA, Halden RU. Bioaccumulation of triclocarban in Lumbriculus variegatus. Environ Toxicol Chem. 2009;28:2580-6.

17. Vollmer G. Disposal of Pharmaceutical Waste in Households - A European Survey. In: Kümmerer K, Hempel M, editors. Kümmerer, Klaus, Hempel, Maximilian, editors. Green and Sustainable Pharmacy. Berlin, Heidelberg: Springer Berlin Heidelberg; 2010. p. 165-78.

18. Costanzo SD, Murby J, Bates J. Ecosystem response to antibiotics entering the aquatic environment. Mar Pollut Bull. 2005;51:218-23.

19. Ziauberyte J. Consumer behaviour regarding household medical waste disposal in Lithuania and Italy. $2011 ;: 68$.

20. National Population Commission. Population and Housing Census of the Federal Republic of Nigeria. Osun State Priority Tables. 2009. http://population.gov.ng/publications/.

21. Krosnick J, Presser S Question and Questionnaire Design. In: Marsden, Peter V. and Wright JD, editor. Handbook of Survey Research. Second Edi. Emerald Group Publishing Limited; 2010. p. 263-314.

22. NAFDAC. Guidelines for handling and disposal of unwholesome medicines and NAFDAC regulated products (food, medicines, medical devices, cosmetics, etc) in Nigeria. Nigeria; 2018.

https://www.nafdac.gov.ng/wp-

content/uploads/Files/Resources/Guidelines/INVESTIGATION_ENFORCEMENT_GUIDELINES/Guidelinesfor-Handling-and-Disposal-of-Unwholesome-Medicines-and-Other-NAFDAC-Regulated-Products.pdf. Accessed 8 Aug 2020.

23. Asa A, Simeon O, David S, Abanishe AH. Unused medicines in Nigerian households: Types and disposal practices. J Pharmacol Pharmacother. 2011;2:195-6.

24. Michael I, Ogbonna B, Sunday N, Anetoh M, Matthew O. Assessment of disposal practices of expired and unused medications among community pharmacies in Anambra State southeast Nigeria: A mixed study design. J Pharm Policy Pract. 2019;12. doi:10.1186/s40545-019-0174-1.

25. Akici A, Aydin V, Kiroglu A. Assessment of the association between drug disposal practices and drug use and storage behaviors. Saudi Pharm J. 2018;26:7-13. doi:10.1016/j.jsps.2017.11.006. 
26. Kahsay H, Ahmedin M, Kebede B, Gebrezihar K, Araya H, Tesfay D. Assessment of Knowledge, Attitude, and Disposal Practice of Unused and Expired Pharmaceuticals in Community of Adigrat City, Northern Ethiopia. J Environ Public Health. 2020;2020:1-11. doi:10.1155/2020/6725423.

27. Ayele Y, Mamu M. Assessment of knowledge, attitude and practice towards disposal of unused and expired pharmaceuticals among community in Harar city, Eastern Ethiopia. J Pharm Policy Pract. 2018;11:27. doi:10.1186/s40545-018-0155-9.

28. Insani WN, Qonita NA, Jannah SS, Nuraliyah NM, Supadmi W, Gatera VA, et al. Improper disposal practice of unused and expired pharmaceutical products in Indonesian households. Heliyon. 2020;6:e04551. doi:10.1016/j.heliyon.2020.e04551.

29. Kumar SL, Logeshwaran LL, Vanitha Rani N, Thennarasu PT, Keerthana MK, Lavanya ML. Assessment of Knowledge and Awareness on the Disposal of Expired and Unused Medicines among Medication Consumers. J Young Pharm. 2019;11:410-6. doi:10.5530/jyp.2019.11.84.

30. Chung S, Brooks BW. Identifying household pharmaceutical waste characteristics and population behaviors in one of the most densely populated global cities. Resour Conserv Recycl. 2019;140:267-77.

31. Leitmeyer K, Adlhoch C. Review Article: Influenza Transmission on Aircraft A Systematic Literature Review. Epidemiology. 2016;27:743-51. doi:10.1097/EDE.0000000000000438.

32. Kelly MP, Barker M. Why is changing health-related behaviour so difficult? Public Health. 2016;136:10916. doi:10.1016/j.puhe.2016.03.030.

33. Ruhoy IS, Daughton CG. Beyond the medicine cabinet: An analysis of where and why medications accumulate. Environ Int. 2008;34:1157-69.

34. Ruhoy IS, Daughton CG. Beyond the medicine cabinet: An analysis of where and why medications accumulate. Environ Int. 2008;34:1157-69. doi:10.1016/j.envint.2008.05.002.

35. Verlicchi P, Galletti A, Petrovic M, Barceló D, Al Aukidy M, Zambello E. Removal of selected pharmaceuticals from domestic wastewater in an activated sludge system followed by a horizontal subsurface flow bed - Analysis of their respective contributions. Sci Total Environ. 2013;454-455:41125. doi:10.1016/j.scitotenv.2013.03.044.

36. Mukattash TL, Alzoubi KH, Abuirjie AM, Jarab AS, Abu Farha RK, Nusair MB, et al. Perceptions and attitudes towards off-label dispensing for pediatric patients, a study of hospital-based pharmacists in Jordan. Saudi Pharm J. 2018;26:20-4. doi:10.1016/j.jsps.2017.11.004.

37. 10.1007/398_2016_3

Paut Kusturica M, Tomas A, Sabo A. Disposal of Unused Drugs: Knowledge and Behavior Among People Around the World. In: Reviews of environmental contamination and toxicology. United States; 2016. p. 71-104. doi:10.1007/398_2016_3.

38. Tong AC, Peake BM, Braund R. Disposal practices for unused medications in New Zealand community pharmacies. J Prim Health Care. 2011;3:197-203.

39. Angi'enda SA, Bukachi SA. Household Knowledge and Perceptions on Disposal Practices of Unused Medicines in Kenya. J Anthropol Archaeol. 2016;4. doi:10.15640/jaa.v4n2a1.

40. Esimai OA, Omoniyi-Esan GO. Wait time and service satisfaction at Antenatal Clinic, Obafemi Awolowo University Ile-Ife. East Afr J Public Health. 2009;6:309-11. 
41. Adamu H, Oche M. Determinants of patient waiting time in the general outpatient department of a tertiary health institution in North-Western Nigeria. Ann Med Health Sci Res. 2013;3:588. doi:10.4103/21419248.122123.

42. Meena. Patients' Response to Waiting Time in an Out-Patient Pharmacy at a Tertiary Care Hospital. J Appl Pharm Sci. 2012;2:090-3. doi:10.7324/JAPS.2012.21018.

43. Abdulkareem I. The surgical waiting time initiative: A review of the Nigerian situation. Niger Med J. 2014;55:443. doi:10.4103/0300-1652.144692.

44. Adindu A, Esu E. Waiting for health care: Clients response to waiting time at the outpatient department of a general hospital in Nigeria. Cont J Trop Med. 2012;6:16-21.

45. Sunny D, Roy K, Benny SS, Mathew DC, Gangadhar Naik J, Gauthaman K. Prescription Audit in an Outpatient Pharmacy of a Tertiary Care Teaching Hospital-A Prospective Study. J Young Pharm. 2019;11:417-20.

46. Grace N, Brian O, Uche M, Valentine U. Assessment of National Health Insurance Scheme's (NHIS) Effectiveness in a Tertiary Teaching Hospital in Southeast Nigeria. J Adv Med Pharm Sci. 2017;13:1-9.

47. Alnahas F, Yeboah P, Fliedel L, Abdin AY, Alhareth K. Expired medication: Societal, regulatory and ethical aspects of a wasted opportunity. International Journal of Environmental Research and Public Health. 2020;17.

48. Sivasankaran P, Mohammed EB, Ganesan N, Durai R. Storage and Safe Disposal of Unwanted/Unused and Expired Medicines: A Descriptive Cross-Sectional Survey among Indian Rural Population. J Young Pharm. 2018.

49. Marina E. Managing Household Pharmaceutical Wastes: The Practices and Perception of Users. 1888; September.

50. Aju-Ameh CO. Mosquitoes are not the major culprits for the high burden of malaria in Nigeria: a commentary. Pan Afr Med J. 2020;35. doi:10.11604/pamj.2020.35.11.16972.

51. Badger-Emeka L. The malaria burden: A look at 3 years outpatient malaria clinic visits in a university community town in Southeast of Nigeria. Niger J Clin Pract. 2020;23:711-9. doi:10.4103/njcp.njcp_218_19.

52. Tong AC, Peake BM, Braund R. Disposal practices for unused medications around the world. Environ Int. 2011;37:292-8. doi:10.1016/j.envint.2010.10.002.

53. Alnahas F, Yeboah P, Fliedel L, Abdin AY, Alhareth K. Expired Medication: Societal, Regulatory and Ethical Aspects of a Wasted Opportunity. Int J Environ Res Public Health. 2020;17:787. doi:10.3390/ijerph17030787.

54. Ariffin M, Zakili TST. Household Pharmaceutical Waste Disposal in Selangor, Malaysia-Policy, Public Perception, and Current Practices. Environ Manage. 2019;64:509-19. doi:10.1007/s00267-019-01199-y.

55. Gold K. Analysis. The Impact of Needle, Syringe, and Lancet Disposal on the Community. J Diabetes Sci Technol. 2011;5:848-50. doi:10.1177/193229681100500404.

56. Obono MO. Evaluation of Pharmaceutical Waste Disposal Practices in Healthcare Facilities in Lagos State, South-West Nigeria. TEXILA Int J PUBLIC Heal. 2016;4:102-11. doi:10.21522/TIJPH.2013.04.04.Art010. 
57. NESREA. Extended producer responsibility programme. 2019. https://www.nesrea.gov.ng/extendedproducer-responsibility/. Accessed 25 Aug 2020.

58. Chung SS, Zheng JS, Burket SR, Brooks BW. Select antibiotics in leachate from closed and active landfills exceed thresholds for antibiotic resistance development. Environ Int. 2018;115:89-96.

59. Massa K, Kilamile F, Safari E, Seleman A, Mwakitalima A, Balengayabo JG, et al. Contributing to the debate on categorising shared sanitation facilities as ' unimproved': An account based on field researchers ' observations and householders ' opinions in three regions, Tanzania. PLoS One. 2017;12:e0185875.

\section{Figures}

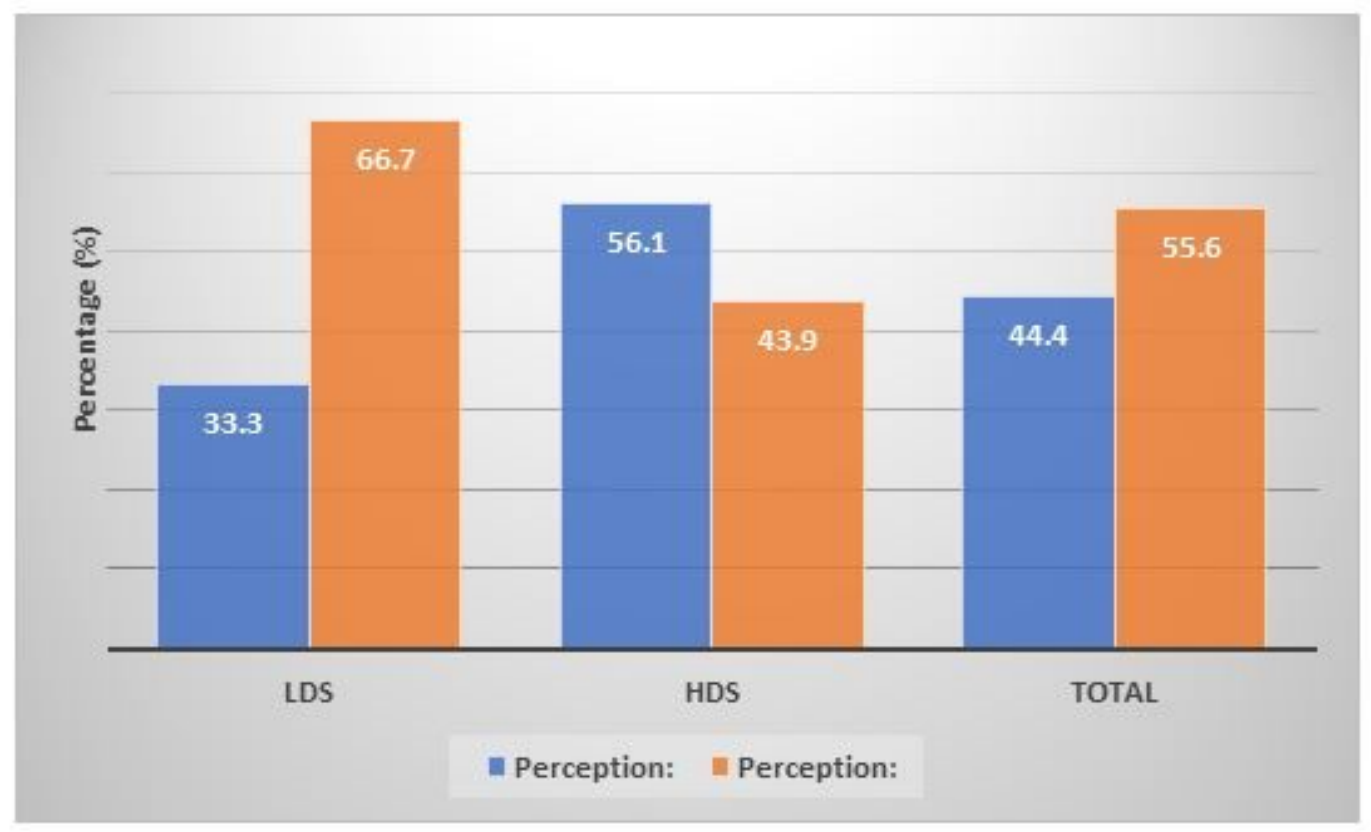

\section{Figure 1}

Perception on disposal of expired and unused medications 


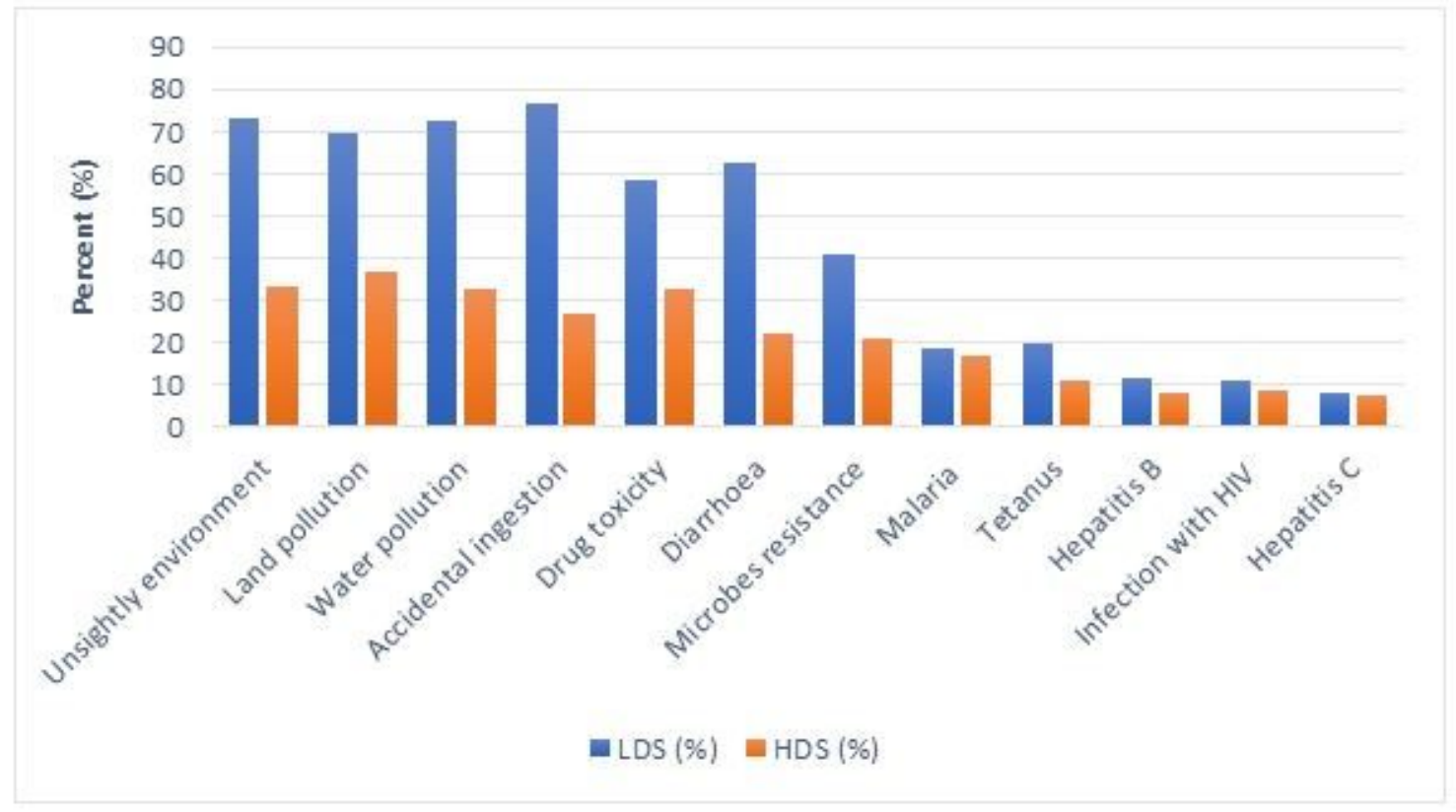

Figure 2

Perceived consequences of indiscriminate disposal of unused and expired medicines 